\title{
Watt Six-Bar Compliant Mechanism Analysis Based on Kinematic and Dynamic Responses
}

\author{
Çağlar Uyulan ${ }^{1, *} \oplus$, Batuhan İpek ${ }^{2} \oplus$ \\ ${ }^{1}$ Dept. of Mechatronics Eng., Bülent Ecevit University, Zonguldak, Turkey \\ 2 Dept. of Mech. Eng., Bülent Ecevit University, Zonguldak, Turkey \\ *Corresponding author: caglaruyulan1@gmail.com
}

Received: 30.04.2021 Accepted: 09.06.2021

\begin{abstract}
In this study, a complete guide to kinematic and kinetic analyses of a Watt type six-bar compliant mechanism is conducted incorporating the flexible buckling of the initially straight element. In the analysis procedure, the hybrid utilization of the pseudo-rigid-body model (PRBM) and the nonlinear elastic theory of beam buckling is presented. This partially compliant mechanism comprises three rigid links and two flexible links. The kinematic analyses of the mechanisms are done by using the vector loop closure equations, the PRBM of a large deflection cantilever beam, and derivation of nonlinear algebraic equations considering the quasi-static equilibrium and load-deflection curve of the flexible parts. Each of the elastic parts makes up a buckling pinned-pinned flexible Euler beam. The vector loop equations are combined with Newton-Euler dynamic formulations to provide the simultaneous constraint matrix. After these operations, the full mechanism is simulated to get both accelerations and forces for each time step. Finally, the design method is validated through experimental results. The findings derived from the combination of buckling elastica solution and PRBM approach enable the analysis of Watt's six-bar compliant mechanism.
\end{abstract}

Keywords: Compliant mechanism; pseudo-rigid-body model; large-deflection analysis; elastica theory; Watt type mechanism

\section{Introduction}

Compliant mechanisms consist of elastic elements that are used to accomplish the desired motion, and compliant mechanisms can have rigid links and flexible links. compliant mechanisms gain their mobility due to the constrained bending of flexible parts from simple topologies (Howell et al., 2013). In compliant mechanisms, force is transferred to other mechanism parts by making use of elastic deformation. The elastic links produce partially or completely the motion of a mechanism (Midha et al., 2000). The popularity of the compliant mechanisms has been increasing during the last decade, because of their superiority and suitability in the micro-electromechanical system (MEMS) design \& micro-and nanofabrication. It is relatively easy to manufacture a compliant mechanism using an injection molding method (Her and Midha, 1987). There are many advantages of compliant mechanisms as compared to rigid-body mechanisms. Integrated functions, high reliability, 
high precision, repeatability, and backlash-free motion, reduced and simplified manufacturing processes, and necessitating fewer parts can also be counted. Therefore, they are suitable to be applied in micro-machining (Khosraviani and Leung, 2012).

They also have some significant challenges. The compliant revolute joints can exhibit small displacements, their mobility is restricted, and cannot sustain continuous rotation. Fatigue failure is observed at the elastic joints. The elastic potential energy is stored due to the deflection of flexible membranes in the compliant mechanism. The strain energy that is stored, is distributed to the entire body and released energy can be used for the desired purpose as a deflection feature. Compliant mechanism performance is affected by material properties, and factors such as thermal and structural loads, and various environment-induced effects (Howell and Midha, 1994).

The compliant mechanisms contribute to many disciplines for countless and versatile engineering solutions (i.e. artificial spinal disc, compliant centrifugal clutch, compliant suspension element, digital micromirrors, piezoresistive pressure sensors). The analysis of the compliant mechanism is quite difficult with large nonlinear elastic deflections with reasonable off-axis stiffness of its flexible elements exist. Their kinematic responses exhibit unique characteristics as compared to the quasi-static response. In synthesizing the compliant mechanisms, the simulation of the mechanism is validated experimentally investigating the complexity of the dynamic relations (Lefebvre et al., 2005; Luo, 2006; Wang and Chen, 2009). The function and structure of mechanisms have been investigated in many studies (Wang et al., 2014; Pieber and Gerstmayr, 2020; Nikham and Farhang, 2018). A method for assisting the design of flexure-based compliant mechanisms for dimensional synthesis, an optimization procedure, and experimental validation has been presented in (Berselli et al., 2015). The mobility of the compliant mechanism was analyzed under the influence of the geometry, material type, and different compliant joints (Pavlovic and Pavlovic, 2005). Realizable joint integration into elastically movable structures for motion tasks was investigated in (Ghosh and Corves, 2015).

Some papers have explained the influence of geometry and material properties on the compliant mechanisms (Gouker et al., 2006; Linß et al., 2019; Jovanova and Frecker, 2017; Venkiteswaran and $\mathrm{Su}, 2016)$. Topology optimization technique to design distributed compliant mechanisms was studied in (Zhang and Zhu, 2018). A comparative study for the synthesis of the design variables of the flexible slider-crank mechanism between PSO and GA was presented (Khemili et al., 2018). The theory of the curves generated in six-link motion has been described (Primrose et al., 1967a; Primrose et al., 19677b). Six-link mechanisms have been used in many applications (i.e., sewing machine (Eren and Aydemir, 2004), prosthetic limbs (Gezgin et al., 2016), legged and jumping robot (Zhang et al., 2020), wheel-track mobile robot (Luo et al., 2018), micro-aerial-vehicle design (Prosser et al., 2011). Design, kinematic synthesis, path, and motion generation with the six-bar linkage mechanism have been investigated in many papers (Chen and Tzeng, 2006; Chen and Huang, 2005; Yi and Leinonen, 2003; Plecnik and Mccarthy, 2014; Zu and Wei, 2013; Mirth and Chase, 1993). A mathematical model of a six-bar linkage parametric design investigated an auto-feeding soup machine. The motion of the mechanism is simulated in ADAMS. The design parameters were also optimized to get a smooth predetermined trajectory, high movement precision, and the smallest extreme acceleration of the soup ladle in motion (Xi et al., 2010).

In another paper, the automated type synthesis of planar mechanisms and multi-body systems were developed by including topology as a design variable in a GA-based methodology. It is possible to design mechanisms that closely match the pre-described kinematic conditions by fusing the GA with a multi-body analysis program (Liu and Mecphee, 2004). A procedure for 
the kinematic synthesis and analysis of a Watt I six-bar linkage was presented to apply in the vehicle suspension design. This approach degrades to the task-based synthesis of a Watt I sixbar where both ground pivots and end effector pivots are particularized (Plecnik et al., 2014). The kinematic performance of the six-bar mechanism used in the prosthetic knee was investigated through the optimization method and the advantages of the six-bar linkage over the four-bar linkage to better achieve the expected trajectory of the ankle joint in the swing phase was revealed (Jin et al., 2003; Bapat and Sujatha, 2017). A software system for the synthesis of spherical Watt I six-bar linkages based on constraining 3R open chain with randomized search in tolerance zones was designed by using SolidWorks Add-In MechGen (Sonawale et al., 2013). The design method of six-bar linkages that reach an over-specified set of task positions explained in (Tsuge and Mccarthy, 2014) expands the number of candidate linkages that approximate an over-specified task. A method of optimization of structural error in a closed kinematic chain of four-bar planar mechanism with six design parameters using the Chebyshev-Freudstein method was applied to increase the global accuracy in the mechanism (Aiswal and Jawale, 2018). In another paper (Mirth, 2012), the GCP to the synthesis of six-bar planar linkages was investigated.

The primary focus of this paper is to design, synthesize and analyze a Watt six-bar compliant mechanism that exploits buckling of pinned-pinned beam elements and large deformed nonlinear beams. Although buckling is usually an unwanted phenomenon, the flexibility of linkages is very useful in applications. Investigating the buckling event can strengthen the compliant mechanism of design space by enabling the flexibility itself for versatile aims. For this purpose, the quasi-static simulation and experimentally validated simulation of the Watt six-bar compliant mechanism response is conducted. Modelling of these mechanisms contributes wide information in several areas, which comprises kinematics, compliant mechanisms, and nonlinear analysis. The sensitivity of the joint clearances and friction are omitted in this paper. The context and content of this paper are as follows. In Section 2.1., a conceptual basis of theories including the theory of elastica and the large-deflection analysis of the cantilever beam is represented by explaining the elliptic integral formulations and polynomial curve fitting application. In Section 2.2., the formulation of the Watt six-bar compliant mechanism, which comprises the vector loop closure method, kinematic analysis, and PRBM, is stated. In Section 2.3., the procedures of the dynamic simulations are investigated considering the simultaneous constraint method and quasi-static analysis, respectively. In Section 3, the simulation and experimental results are presented. The concluding remarks and discussions are given in Section 4 .

\section{Experimental Methods}

\subsection{The Theoretical Basis}

Elastica theory predicts the movement of the large deflecting beams incorporated in the compliant mechanisms and is utilized in compliant mechanism design and synthesis (Howell and Midha, 1994; Konopasek, 1980). Jacob Bernoulli has pioneered the large-deflection-based analysis by proposing the proportional relation between the beam curvature at any point and bending moment, and Euler solved elastica problems in a closed-form. The main restriction is that it is only valid for simple geometries and simple loading conditions. The large deflection equations of the pinned-pinned buckling mounted to a slider can be stated in normalized form as in Eq. (1) (Sönmez and Tutum,2008; Sönmez, 2000). 


$$
\begin{gathered}
-\left(p^{2}+q^{2}\right)^{0.25} \pm 2 F(\chi, \varphi)=0 \\
-w\left(p^{2}+q^{2}\right)^{0.75} \pm p(4 E(\chi, \varphi)-2 F(\chi, \varphi))=0 \\
-h\left(p^{2}+q^{2}\right)^{0.75} \pm q(4 E(\chi, \varphi)-2 F(\chi, \varphi))=0,
\end{gathered}
$$

where $p=\frac{P L^{2}}{E I}$ is the normalized load and $q=\frac{Q L^{2}}{E I}$ is the normalized deflection. $P$ is the horizontal force, $Q$ is the vertical reaction force applied on the beam by the slider. $w=\frac{W}{L}, h=$ $\frac{H}{L}$ are normalized flexible beam horizontal displacement and vertical offset, respectively. $L$ is the length of the compliant beam.

In Eq. (1), $F(\chi, \varphi)$ and $E(\chi, \varphi)$ are completely first and second kind of elliptic integrals, respectively. $\varphi=\frac{\pi}{2}$ and $\chi$ are the amplitude and the modulus. The formulation is represented in Eq. (2):

$$
\begin{gathered}
F(\chi, \varphi)=\int_{0}^{\varphi} \frac{d \varphi}{\sqrt{1-\chi^{2} \sin ^{2}(\varphi)}} \\
E(\chi, \varphi)=\int_{0}^{\varphi} \sqrt{1-\chi^{2} \sin ^{2}(\varphi) d \varphi}
\end{gathered}
$$

Equation (2) is solved to evaluate the nonlinear load-deflection behaviour of a pinned-pinned beam subjected to a horizontal load. The forces exerted to a simple pinned-pinned section are collinear along the line among the two-pin joints and the deflections are also along this line. The moments are not carried by pin ends. A nonlinear spring defined with a polynomial loaddeflection equation defines the pin-pin segment model.

To conduct the large deflection analysis, assume an initially straight cantilever beam subjected to an end force having a vertical force component $(Q)$ along the positive $y$-axis and a horizontal force component $(n Q)$ along the negative $\mathrm{x}$-axis, as demonstrated in Figure 1.

a)

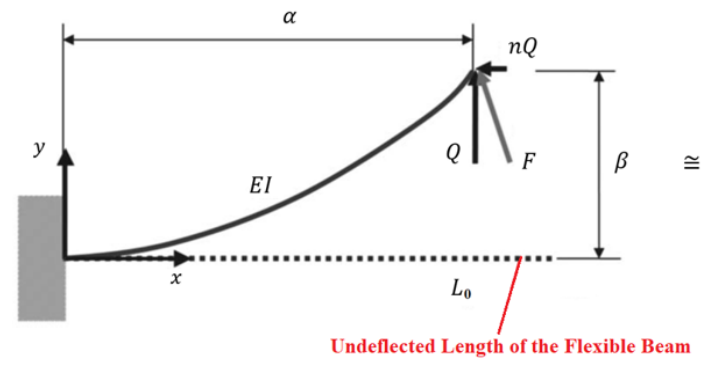

b)

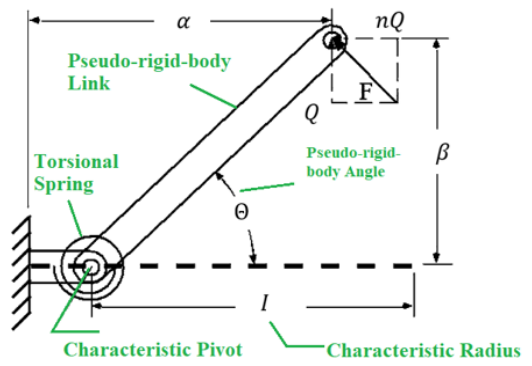

Figure 1. The demonstration of the large deflecting cantilever beam subjected a force at the free end; a) fixed-pinned segment, b) its equivalent pseudo-rigid-body model.

The parameter $n$ is the force coefficient, which implies the proportion of the horizontal force to the vertical force, and positive if the beam is compressed. The parameters presented in Figure 1 are also modelled considering elliptic integrals in Eq. (3). The reader should refer to (Zhang and Chen, 2012; Bishopp and Drucker, 1945), or (Sönmez and Tutum, 2008) for their derivation. 


$$
\begin{gathered}
\alpha=\frac{1}{\sqrt{\eta}}(F(t, \varphi)-F(\gamma, t)) \text { for } \theta_{0}<\varphi_{1} \\
\frac{\alpha}{l}=\frac{1}{\varpi \eta^{5 / 2}}[-n \eta((F(t, \varphi)-F(\gamma, t)+2 E(\gamma, t)-2 E(t, \varphi))+\sqrt{2 \eta(\eta+\lambda)} \cos (\gamma)] \\
\frac{\beta}{l}=\frac{1}{\varpi \eta^{5 / 2}}[\eta(F(t, \varphi)-F(\gamma, t)+2 E(\gamma, t)-2 E(t, \varphi))+n \sqrt{2 \eta(\eta+\lambda)} \cos (\gamma)] \\
\eta=\sqrt{1+n^{2}}, \quad \gamma=\arcsin \left(\sqrt{\frac{\eta-n}{\eta+\lambda}}\right), \quad t=\sqrt{\frac{\eta+\lambda}{2 \eta}}
\end{gathered}
$$

where $\alpha$ is the horizontal position, $\beta$ is the vertical deflection of the tip of the beam, $\lambda=$ $\sin \left(\theta_{0}\right)-n \cos \left(\theta_{0}\right)$ is an end angle related parameter, $\theta_{0}$ is the deflected beam end angle, and $\varphi_{1}=\arctan \left(\frac{1}{n}\right)$ is the exerted load angle accepted positive from the $x$-axis. $\varpi=\frac{Q L_{0}^{2}}{E I}$ is the corresponding loading parameter. The exact elastica solution of the flexible beams and its trajectories are used for the kineostatic analysis simulation results.

\subsection{Formulation of Watt Six-Bar Compliant Mechanism}

The concept of the vector closed-loop equation (or loop closure equation) is presented in Figure 2.

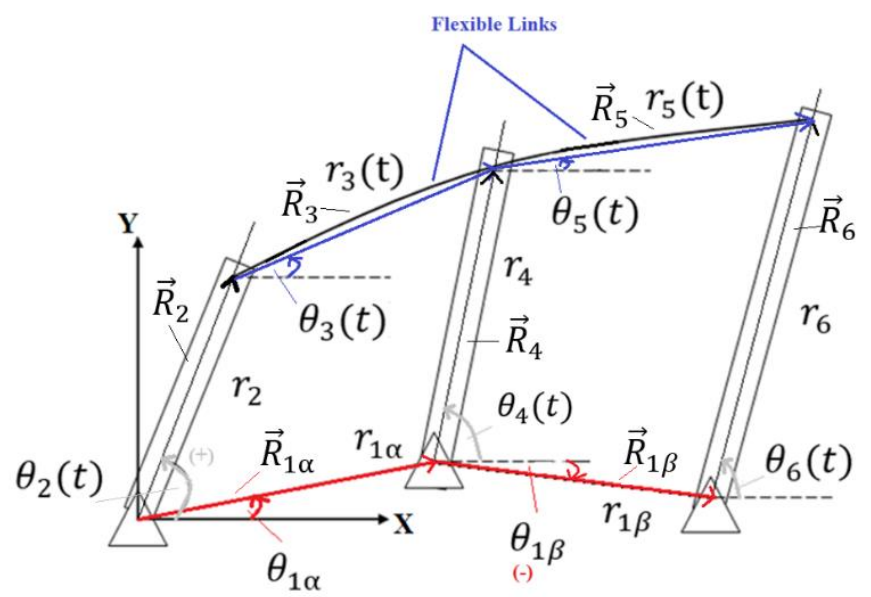

Figure 2. Parameterization of the dimensions and conceptualization of vector loop closure. Note that the angles of the vectors (measured from the positive $x$-axis to the vector at its tail) are the same as the corresponding link angle. The angles are defined as counterclockwise positive from the positive $\mathrm{x}$-direction and drawn to the tail of each vector.

As seen in Figure 2, arbitrary coordinates are assigned at the ground joint, which is the leftmost in the figure. Each link (including the ground links) is represented by a displacement vector. The closed-loop vector equations are stated in Eq. (4):

$$
\begin{aligned}
& \vec{R}_{2}+\vec{R}_{3}=\vec{R}_{1 \alpha}+\vec{R}_{4} \\
& \vec{R}_{4}+\vec{R}_{5}=\vec{R}_{1 \beta}+\vec{R}_{6}
\end{aligned}
$$

The vector loop equation is a very compact expression of the constraints that exist between the various bodies of mechanism. The displacement achieved when vectors at the LHS of the equation are added is the same as that achieved when vectors at the RHS of the equation are added. This vector loop equation must be satisfied by the mechanism, regardless of the pose, 
it takes, as long as it remains assembled as a mechanism. Eq. (5) is obtained by summing independently the horizontal (x-direction) and vertical (y-direction) vector components.

$$
\begin{aligned}
& r_{2} \cos \left(\theta_{2}(t)\right)+r_{3}(\mathrm{t}) \cos \left(\theta_{3}(t)\right)=r_{1 \alpha} \cos \left(\theta_{1 \alpha}\right)+r_{4} \cos \left(\theta_{4}(t)\right) \\
& r_{2} \sin \left(\theta_{2}(t)\right)+r_{3}(\mathrm{t}) \sin \left(\theta_{3}(t)\right)=r_{1 \alpha} \sin \left(\theta_{1 \alpha}\right)+r_{4} \sin \left(\theta_{4}(t)\right) \\
& r_{4} \cos \left(\theta_{4}(t)\right)+r_{5}(\mathrm{t}) \cos \left(\theta_{5}(t)\right)=r_{1 \beta} \cos \left(\theta_{1 \beta}\right)+r_{6} \cos \left(\theta_{6}(t)\right) \\
& r_{4} \sin \left(\theta_{4}(t)\right)+r_{5}(\mathrm{t}) \sin \left(\theta_{5}(t)\right)=r_{1 \beta} \sin \left(\theta_{1 \beta}\right)+r_{6} \sin \left(\theta_{6}(t)\right)
\end{aligned}
$$

The derived vector loop equations are compact, elegant expressions of the simple fact the various links of the mechanism are constrained in some manner. The evaluations of the link velocities and accelerations can be obtained by taking the first and second derivatives w.r.t time of the Eq. (5), respectively. The vectors are time-varying and their orientation (vector direction) changes as the mechanism moves. Due to the flexible structure of the link-3 and link-5, their magnitude of link lengths also changes while the other link lengths (link-2, link-4, link-6) remain constant. There are seven link angles, two of which $\left(\theta_{1 \alpha}, \theta_{1 \beta}\right)$ can be taken to be constant. The other five angles are time-varying, and that must be taken into account when taking the derivatives. Eq. (6), which specifies the relationship among the rotational rates of the moving links as long as the mechanism remains assembled.

$$
\begin{aligned}
& r_{2} \dot{\theta}_{2}(t) \sin \left(\theta_{2}(t)\right)+r_{3}(\mathrm{t}) \dot{\theta}_{3}(t) \sin \left(\theta_{3}(t)\right)-\dot{r}_{3}(\mathrm{t}) \cos \left(\theta_{3}(t)\right)=r_{4} \dot{\theta}_{4}(t) \sin \left(\theta_{4}(t)\right) \\
& r_{2} \dot{\theta}_{2}(t) \cos \left(\theta_{2}(t)\right)+\dot{r}_{3}(\mathrm{t}) \sin \left(\theta_{3}(t)\right)+r_{3}(\mathrm{t}) \dot{\theta}_{3}(t) \cos \left(\theta_{3}(t)\right)=r_{4} \dot{\theta}_{4}(t) \cos \left(\theta_{4}(t)\right) \\
& r_{4} \dot{\theta}_{4}(t) \sin \left(\theta_{4}(t)\right)+r_{5}(\mathrm{t}) \dot{\theta}_{5}(t) \sin \left(\theta_{5}(t)\right)-\dot{r}_{5}(\mathrm{t}) \cos \left(\theta_{5}(t)\right)=r_{6} \dot{\theta}_{6}(t) \sin \left(\theta_{6}(t)\right) \\
& r_{4} \dot{\theta}_{4}(t) \cos \left(\theta_{4}(t)\right)+\dot{r}_{5}(\mathrm{t}) \sin \left(\theta_{5}(t)\right)+r_{5}(\mathrm{t}) \dot{\theta}_{5}(t) \cos \left(\theta_{5}(t)\right)=r_{6} \dot{\theta}_{6}(t) \cos \left(\theta_{6}(t)\right)
\end{aligned}
$$

Link-2 is attached to a large DC motor that is capable of providing enough torque. Under this condition, $\dot{\theta}_{2}(t)$ is the input to the mechanism. The second derivative of the loop equation is obtained in Eq. (7):

$$
\begin{gathered}
\ddot{r}_{3}(t) \cos \left(\theta_{3}(t)\right)+r_{4} \dot{\theta}_{4}(t)^{2} \cos \left(\theta_{4}(t)\right)+r_{4} \ddot{\theta}_{4}(t) \sin \left(\theta_{4}(t)\right) \\
=2 \dot{r}_{3}(\mathrm{t}) \dot{\theta}_{3}(t) \sin \left(\theta_{3}(t)\right)+r_{2} \dot{\theta}_{2}(t)^{2} \cos \left(\theta_{2}(t)\right)+r_{2} \ddot{\theta}_{2}(t) \sin \left(\theta_{2}(t)\right) \\
+r_{3}(\mathrm{t}) \dot{\theta}_{3}(t)^{2} \cos \left(\theta_{3}(t)\right)+r_{3}(\mathrm{t}) \ddot{\theta}_{3}(t) \sin \left(\theta_{3}(t)\right) \\
\ddot{r}_{3}(t) \sin \left(\theta_{3}(t)\right)+2 \dot{r}_{3}(\mathrm{t}) \dot{\theta}_{3}(t) \cos \left(\theta_{3}(t)\right)+r_{4} \dot{\theta}_{4}(t)^{2} \sin \left(\theta_{4}(t)\right) \\
\quad+r_{2} \ddot{\theta}_{2}(t) \cos \left(\theta_{2}(t)\right)+r_{3}(\mathrm{t}) \ddot{\theta}_{3}(t) \cos \left(\theta_{3}(t)\right) \\
=r_{2} \dot{\theta}_{2}(t)^{2} \sin \left(\theta_{2}(t)\right)+r_{4} \ddot{\theta}_{4}(t) \cos \left(\theta_{4}(t)\right)+r_{3}(\mathrm{t}) \dot{\theta}_{3}(t)^{2} \sin \left(\theta_{3}(t)\right) \\
\ddot{r}_{5}(t) \cos \left(\theta_{5}(t)\right)+r_{6} \dot{\theta}_{6}(t)^{2} \cos \left(\theta_{6}(t)\right)+r_{6} \ddot{\theta}_{6}(t) \sin \left(\theta_{6}(t)\right) \\
=2 \dot{r}_{5}(\mathrm{t}) \dot{\theta}_{5}(t) \sin \left(\theta_{5}(t)\right)+r_{4} \dot{\theta}_{4}(t)^{2} \cos \left(\theta_{4}(t)\right)+r_{4} \ddot{\theta}_{4}(t) \sin \left(\theta_{4}(t)\right) \\
\quad+r_{5}(\mathrm{t}) \dot{\theta}_{5}(t)^{2} \cos \left(\theta_{5}(t)\right)+r_{5}(\mathrm{t}) \ddot{\theta}_{5}(t) \sin \left(\theta_{5}(t)\right) \\
\dot{\theta}_{5}(t) \sin \left(\theta_{5}(t)\right)+2 \dot{r}_{5}(\mathrm{t}) \dot{\theta}_{5}(t) \cos \left(\theta_{5}(t)\right)+r_{6} \dot{\theta}_{6}(t)^{2} \sin \left(\theta_{6}(t)\right) \\
\quad+r_{4} \ddot{\theta}_{4}(t) \cos \left(\theta_{4}(t)\right)+r_{5}(\mathrm{t}) \ddot{\theta}_{5}(t) \cos \left(\theta_{5}(t)\right) \\
=r_{4} \dot{\theta}_{4}(t)^{2} \sin \left(\theta_{4}(t)\right)+r_{6} \ddot{\theta}_{6}(t) \cos \left(\theta_{6}(t)\right)+r_{5}(\mathrm{t}) \dot{\theta}_{5}(t)^{2} \sin \left(\theta_{5}(t)\right)
\end{gathered}
$$

PRBM concept analyses large beam deflections as rigid links with pin joints and torsional springs. PRBM's have been classified based on various types of compliant segments (i.e. small- 
length flexural pivots, fixed-pinned segments, fixed-guided parts, binary pinned-pinned components, functionally binary pinned-pinned segments). PRBM simplifies the nonlinear analysis of the flexible beam elements by reducing the exact solution time while keeping the accuracy high. The reduced-order approach is essential for optimization and control. The PRBM consists of a rigid-body mechanism tracking trajectory with the tip of the original flexible beam and a nonlinear spring, which gives the same angular deflection (Yu et al., 2018; Ramirez and Lusk, 2011; Luo et al., 2015).

The deflections can be modelled by two rigid links pinned at the centre of the circular path. Pivot location is defined by the nondimensional "characteristic radius factor $(\gamma-$ Figure 1$)$. The length of the pseudo-rigid link is $r_{i}=L_{i}+\varepsilon l_{i}=\gamma l_{i}$, where $l_{i}$ is the flexible segment length ("characteristic radius-Figure 1") and $L_{i}$ is the length of the rigid part. Flexible beam deflections are obtained as $\alpha=l_{i}(1-\gamma) \cos (\Theta), \beta=l_{i} \gamma \sin (\Theta)$.

Deflection resistance is modelled by a torsional spring with a torsional spring constant as in Eq. (8):

$$
K_{i}=\gamma K_{\Theta, i} \frac{E I_{i}}{l_{i}}
$$

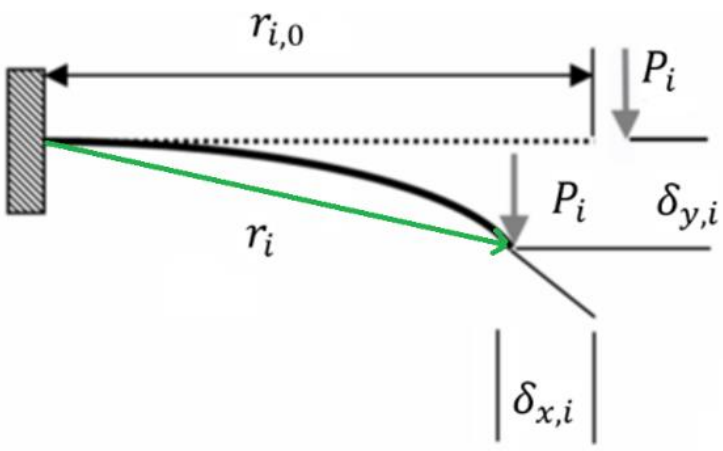

Figure 3. Cantilever beam subject to an end load.

Formulas used for nonlinear large deflections of pinned-pinned flexible link depicted in Figure 3 are stated as in Eq. (9):

$$
\begin{gathered}
\vartheta_{i}(t)=\delta_{y, i}(t) r_{i, 0}=\left(r_{i, 0}-r_{i}(t)\right) \\
I_{c i}=\frac{1}{12} h_{f, i}\left(b_{f, i}\right)^{3} \\
F_{i}(t)=\frac{E I_{i}}{\left(r_{i, 0}\right)^{2}} P_{i}(t),
\end{gathered}
$$

where $\delta_{y, i}$ and $P_{i}$ are normalized deflection and load matrix, respectively.

The forces produced by flexible links are modelled using $4^{\text {th }}$ order polynomial functions derived from nonlinear inextensible exact elastic theory. $F_{i}(t)$ can be approximated with a $4^{\text {th }}$ order polynomial function of deflection given in Eq. (10):

$$
F_{i}(t)=K_{i, 5}\left(\vartheta_{i}(t)\right)^{4}+K_{i, 4}\left(\vartheta_{i}(t)\right)^{3}+K_{i, 3}\left(\vartheta_{i}(t)\right)^{2}+K_{i, 2} \vartheta_{i}(t)+K_{i, 1}
$$

The force-deflection curve of the flexible joints are plotted in Figure 4. 


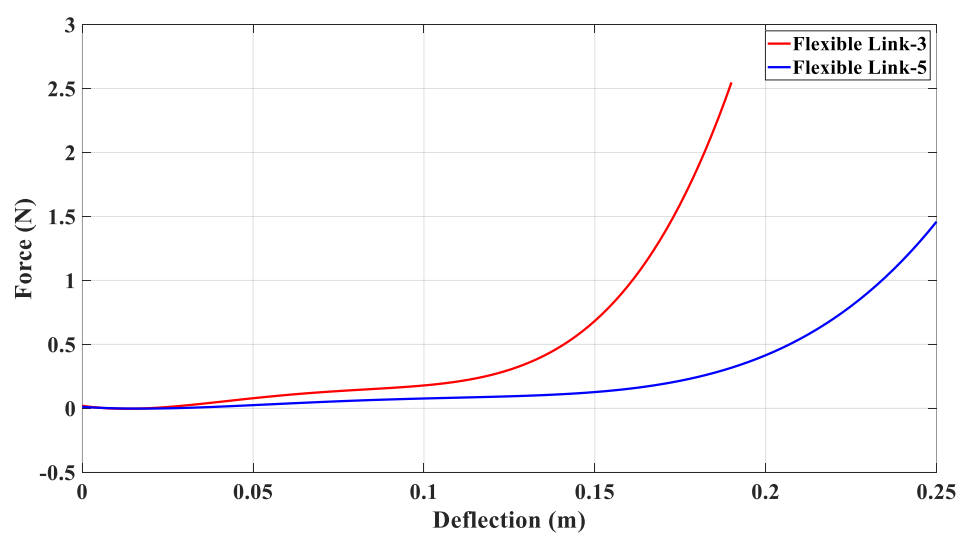

Figure 4. The force-deflection curves of the flexible joints. the pin-pin beam buckling; derived from the solution of the exact elastica.

The coefficients of the polynomial function are evaluated from the flexible links forcedeflection curve through the curve fitting algorithm. The values of the coefficients are presented in Table 1.

Table 1. Curve-fitted coefficients of the flexible joints.

\begin{tabular}{|c|c|}
\hline$K_{3,5}$ & 9673 \\
\hline$K_{3,4}$ & -2418 \\
\hline$K_{3,3}$ & 201.7 \\
\hline$K_{3,2}$ & -4.078 \\
\hline$K_{3,1}$ & 0.02046 \\
\hline$K_{5,5}$ & 1864 \\
\hline$K_{5,4}$ & -613.1 \\
\hline$K_{5,3}$ & 67.09 \\
\hline$K_{5,2}$ & -1.79 \\
\hline$K_{5,1}$ & 0.01182 \\
\hline
\end{tabular}

\subsection{Dynamic Simulation via Simultaneous Constraint Method}

The simultaneous constraint method builds on the kinematic relationships described in this section. The second derivatives of the loop equation are obtained. Then, simple force balances are applied to each link to relate the forces on each link to its acceleration. Next, an approach similar to the vector loop methodology yields additional information on the link acceleration of the centre of mass. The equations are combined into a sparse matrix that can be evaluated through MATLAB/SIMULINK as a component of a full simulation. The free-body diagrams (FBD's) of the components of the Watt six-bar compliant mechanism drawn in Solidworks are represented in Figure 5. 

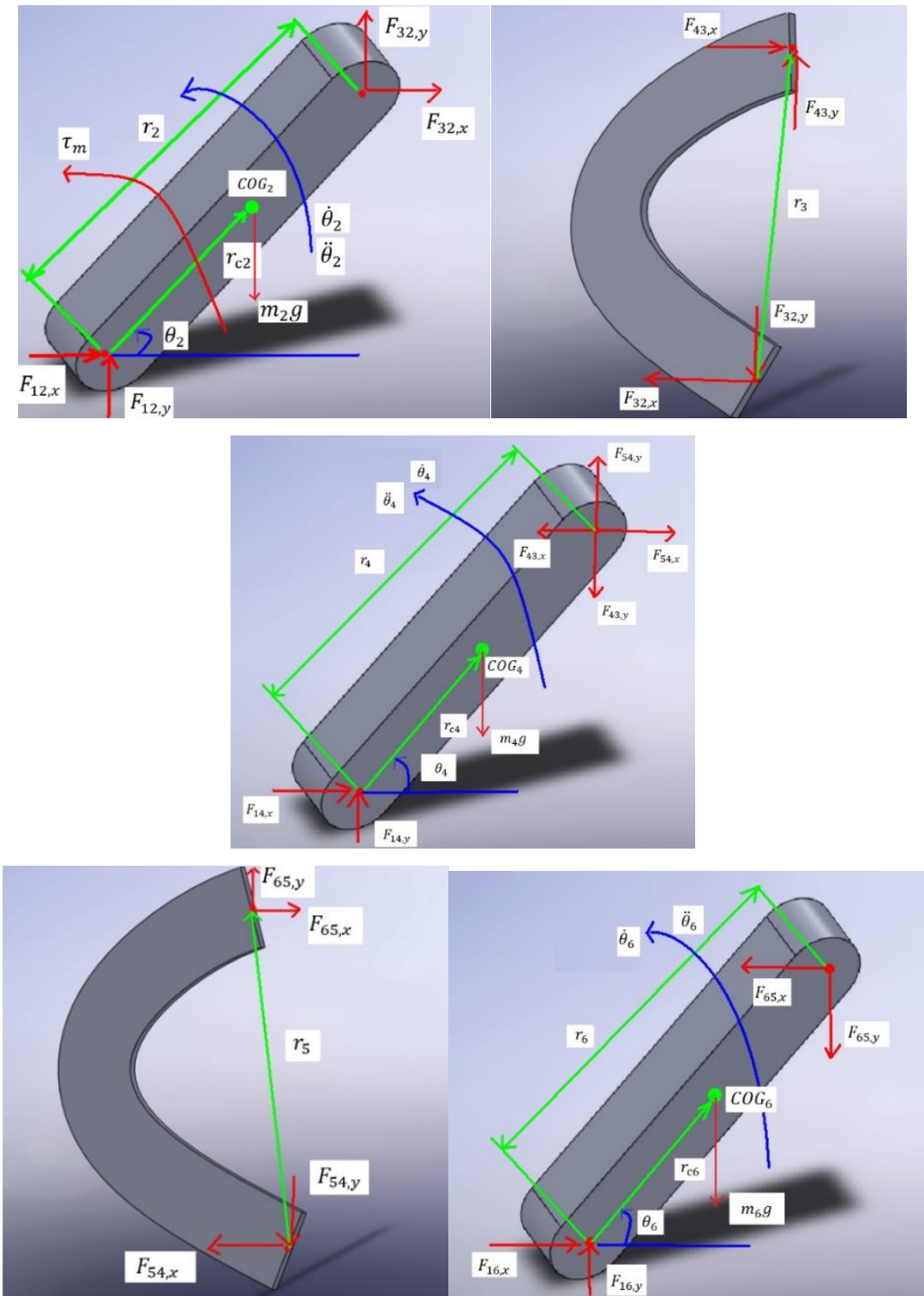

Figure 5. FBD's of link-2, link-3, link-4, link-5, link-6, respectively.

\subsubsection{Dynamic Equations}

Each joint can sustain only a force and that the forces are broken down into $\mathrm{x}$-and $\mathrm{y}$ components. In general, $\vec{F}_{i j}$ will be for the force exerting on the link $j$, originating from the link $i$. An essential point here is that $\vec{F}_{j i}$ is equal and opposite $\vec{F}_{i j}$. Force equations are the result of Newton's Second Law, applied to the links.

The equations of motion (EOM) for the $i^{\text {th }}$ link can be written as in Eq. (11).

$$
\begin{gathered}
F_{j i, x}+F_{k i, x}=m_{i} a_{c i, x} \\
F_{j i, y}+F_{k i, y}-m_{i} g=m_{i} a_{c i, y}
\end{gathered}
$$


where the acceleration terms $\left(a_{c i, x}, a_{c i, y}\right)$ are the $\mathrm{x}$ - and y-projections of the acceleration of the centre of mass of the link. Those accelerations are hidden in the vector loop equations for the entire mechanism. For each link, another position vector equation should be derived that maps the acceleration of the COG of each link to the other state variables.

The vector relationships to derive the acceleration of the centre of mass of links are given in Eq. (12):

$$
\begin{aligned}
& a_{c 2, x}(t)=-r_{c 2} \dot{\theta}_{2}(t)^{2} \cos \left(\theta_{2}(t)\right)-r_{c 2} \ddot{\theta}_{2}(t) \sin \left(\theta_{2}(t)\right) \\
& a_{c 2, y}(t)=-r_{c 2} \dot{\theta}_{2}(t)^{2} \sin \left(\theta_{2}(t)\right)+r_{c 2} \ddot{\theta}_{2}(t) \cos \left(\theta_{2}(t)\right) \\
& a_{c 4, x}(t)=-r_{c 4} \dot{\theta}_{4}(t)^{2} \cos \left(\theta_{4}(t)\right)-r_{c 4} \ddot{\theta}_{4}(t) \sin \left(\theta_{4}(t)\right) \\
& a_{c 4, y}(t)=-r_{c 4} \dot{\theta}_{4}(t)^{2} \sin \left(\theta_{4}(t)\right)+r_{c 4} \ddot{\theta}_{4}(t) \cos \left(\theta_{4}(t)\right) \\
& a_{c 6, x}(t)=-r_{c 6} \dot{\theta}_{6}(t)^{2} \cos \left(\theta_{6}(t)\right)-r_{c 6} \ddot{\theta}_{6}(t) \sin \left(\theta_{6}(t)\right) \\
& a_{c 6, y}(t)=-r_{c 6} \dot{\theta}_{6}(t)^{2} \sin \left(\theta_{6}(t)\right)+r_{c 6} \ddot{\theta}_{6}(t) \cos \left(\theta_{6}(t)\right)
\end{aligned}
$$

The general form of the relationships between the summation of moments and the angular acceleration can be reduced by choosing the centre of gravity $\left(C O G_{j}\right)$ of the links as an axis about which the moments are taken. The summation of moments leads to Eq. (13):

$$
F_{i j, x} r_{c i} \sin \left(\theta_{i}\right)-F_{i j, y} r_{c i} \cos \left(\theta_{i}\right)-F_{k i, x}\left(r_{i}-r_{c i}\right) \sin \left(\theta_{i}\right)+F_{k i, y}\left(r_{i}-r_{c i}\right) \cos \left(\theta_{i}\right)=I_{c i} \ddot{\theta}_{i}
$$

After all, the force and moment equilibrium of the rigid links is built as in Eq. (14):

$$
\begin{gathered}
F_{12, x}(t)+F_{3}(\mathrm{t}) \cos \left(\theta_{3}(t)\right)=m_{2} a_{c 2, x}(t) \\
F_{12, y}(t)+F_{3}(\mathrm{t}) \sin \left(\theta_{3}(t)\right)-m_{2} g=m_{2} a_{c 2, y}(t) \\
\tau_{m}(t)+F_{12, x}(t) r_{c 2} \sin \left(\theta_{2}(t)\right)-F_{12, y}(t) r_{c 2} \cos \left(\theta_{2}(t)\right) \\
-F_{3}(t) \cos \left(\theta_{3}(t)\right)\left(r_{2}-r_{c 2}\right) \sin \left(\theta_{2}(t)\right)+F_{3}(\mathrm{t}) \sin \left(\theta_{3}(t)\right)\left(r_{2}-r_{c 2}\right) \cos \left(\theta_{2}(t)\right)=I_{c 2} \ddot{\theta}_{2}(t) \\
F_{14, x}(t)+F_{5}(\mathrm{t}) \cos \left(\theta_{5}(t)\right)+F_{3}(\mathrm{t}) \cos \left(\theta_{3}(t)\right)=m_{4} a_{c 4, x}(t) \\
F_{14, y}(t)+F_{5}(\mathrm{t}) \sin \left(\theta_{5}(t)\right)+F_{3}(\mathrm{t}) \sin \left(\theta_{3}(t)\right)-m_{4} g=m_{4} a_{c 4, y}(t) \\
F_{14, x}(t) r_{c 4} \sin \left(\theta_{4}(t)\right)-F_{14, y}(t) r_{c 4} \cos \left(\theta_{4}(t)\right) \\
-F_{5}(t) \cos \left(\theta_{5}(t)\right)\left(r_{4}-r_{c 4}\right) \sin \left(\theta_{4}(t)\right) \\
+F_{5}(\mathrm{t}) \sin \left(\theta_{5}(t)\right)\left(r_{4}-r_{c 4}\right) \cos \left(\theta_{4}(t)\right) \\
-F_{3}(t) \cos \left(\theta_{3}(t)\right)\left(r_{4}-r_{c 4}\right) \sin \left(\theta_{4}(t)\right) \\
+F_{3}(t) \sin \left(\theta_{3}(t)\right)\left(r_{4}-r_{c 4}\right) \cos \left(\theta_{4}(t)\right)=I_{c 4} \ddot{\theta}_{4}(t) \\
F_{16, x}(t)+F_{5}(\mathrm{t}) \cos \left(\theta_{5}(t)\right)=m_{6} a_{c 6, x}(t) \\
F_{16, y}(t)+F_{5}(\mathrm{t}) \sin \left(\theta_{5}(t)\right)-m_{6} g=m_{6} a_{c 6, y}(t) \\
F_{16, x}(t) r_{c 6} \sin \left(\theta_{6}(t)\right)-F_{16, y}(t) r_{c 6} \cos \left(\theta_{6}(t)\right) \\
-F_{5}(t) \cos \left(\theta_{5}(t)\right)\left(r_{6}-r_{c 6}\right) \sin \left(\theta_{6}(t)\right) \\
+F_{5}(\mathrm{t}) \sin \left(\theta_{5}(t)\right)\left(r_{6}-r_{c 6}\right) \cos \left(\theta_{6}(t)\right)=I_{c 6} \ddot{\theta}_{6}(t)
\end{gathered}
$$




\subsubsection{Solution Procedure of the Flexible Pin-Pin Large Deflection}

The deflections of the elastic links are calculated by using quasi-static analysis (Lobontiu, 2014) while the mechanism moves. The simulation of the mechanism is conducted under the condition of quasi-statically slow speeds while omitting the beam and joints inertial forces\&damping forces. System modelling and simulation can be made by adding the inertia and the damping forces. When the mass of the flexible beam is small compared to the rigid parts, it may not be included in the inertia of the system. The inertia of the flexible moving parts might then be modelled as a lumped mass. Newton-Raphson method is utilized to reveal this equation set starting from the initially compressed state of the mechanism. As the link-2 turns quasi-statically through the full cycle of $360^{\circ}$ in the reverse direction, the solution for the previous step is utilized as the initial guess for the next iteration The unknowns $r_{3}(\mathrm{t}), \theta_{3}(t)$, $r_{5}(\mathrm{t}), \theta_{5}(t), \theta_{4}(t), \theta_{6}(t)$, and $\tau_{m}(t)$ are evaluated after solving Eq. (15) simultaneously.

$$
\begin{gathered}
r_{2} \cos \left(\theta_{2}(t)\right)+r_{3}(\mathrm{t}) \cos \left(\theta_{3}(t)\right)-r_{1 \alpha} \cos \left(\theta_{1 \alpha}\right)-r_{4} \cos \left(\theta_{4}(t)\right)=0 \\
r_{2} \sin \left(\theta_{2}(t)\right)+r_{3}(\mathrm{t}) \sin \left(\theta_{3}(t)\right)-r_{1 \alpha} \sin \left(\theta_{1 \alpha}\right)-r_{4} \sin \left(\theta_{4}(t)\right)=0 \\
r_{4} \cos \left(\theta_{4}(t)\right)+r_{5}(\mathrm{t}) \cos \left(\theta_{5}(t)\right)-r_{1 \beta} \cos \left(\theta_{1 \beta}\right)-r_{6} \cos \left(\theta_{6}(t)\right)=0 \\
r_{4} \sin \left(\theta_{4}(t)\right)+r_{5}(\mathrm{t}) \sin \left(\theta_{5}(t)\right)-r_{1 \beta} \sin \left(\theta_{1 \beta}\right)-r_{6} \sin \left(\theta_{6}(t)\right)=0 \\
\tau_{m}(t)+F_{3}(\mathrm{t}) \sin \left(\theta_{3}(t)\right)\left(r_{2}\right) \cos \left(\theta_{2}(t)\right)-F_{3}(t) \cos \left(\theta_{3}(t)\right)\left(r_{2}\right) \sin \left(\theta_{2}(t)\right)=0 \\
-F_{5}(t) \cos \left(\theta_{5}(t)\right)\left(r_{4}\right) \sin \left(\theta_{4}(t)\right) \\
+F_{5}(\mathrm{t}) \sin \left(\theta_{5}(t)\right)\left(r_{4}\right) \cos \left(\theta_{4}(t)\right)-F_{3}(t) \cos \left(\theta_{3}(t)\right)\left(r_{4}\right) \sin \left(\theta_{4}(t)\right) \\
\quad+F_{3}(t) \sin \left(\theta_{3}(t)\right)\left(r_{4}\right) \cos \left(\theta_{4}(t)\right)=0 \\
-F_{5}(t) \cos \left(\theta_{5}(t)\right)\left(r_{6}\right) \sin \left(\theta_{6}(t)\right)+F_{5}(\mathrm{t}) \sin \left(\theta_{5}(t)\right)\left(r_{6}\right) \cos \left(\theta_{6}(t)\right)=0
\end{gathered}
$$

To solve these nonlinear equations, one should develop a numerical method having proper initial conditions. The mechanism is simulated by using Matlab codes that contain "lsqnonlin" command with options [options = optimset('maxfunevals',3000000,'maxiter', 400000,'TolFun',1e-20,'TolX',1e-20)], and trust-region-reflective-algorithm.

For kinematic analysis, $r_{3}(\mathrm{t})$ and $r_{5}(\mathrm{t})$ elastic link deflections must be known. At this point, the results of the quasi-static analysis help us to calculate elastic link deflections and also reduce the number of unknown equations. Nonlinear load-deflection analysis of the flexible beam is represented by a polynomial fitted by using nonlinear inextensible exact elastic theory. $\theta_{2}-r_{3}$ and $\theta_{2}-r_{5}$ curves represented in Figure 6 obtained from quasi-static results. 
(a)

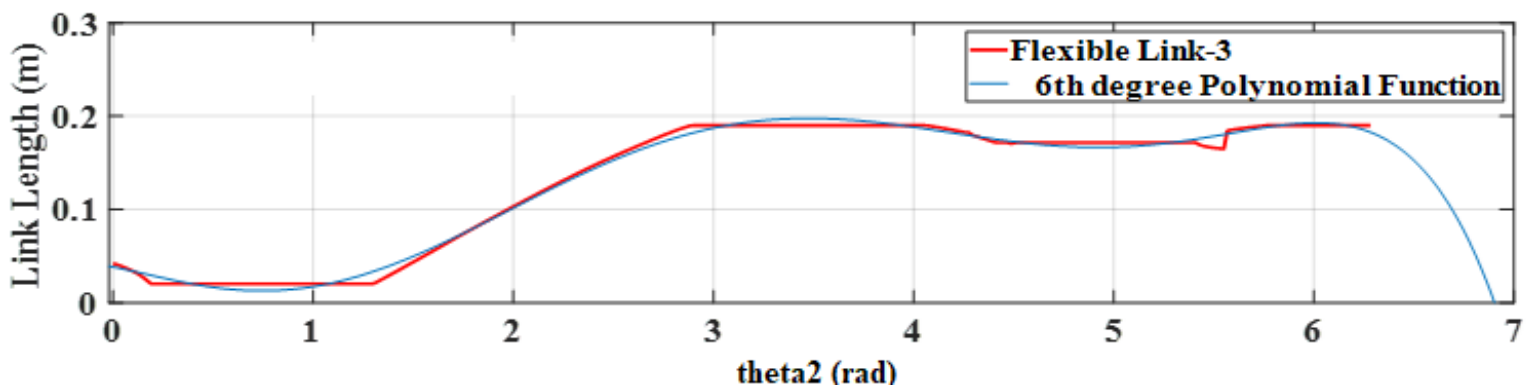

b)

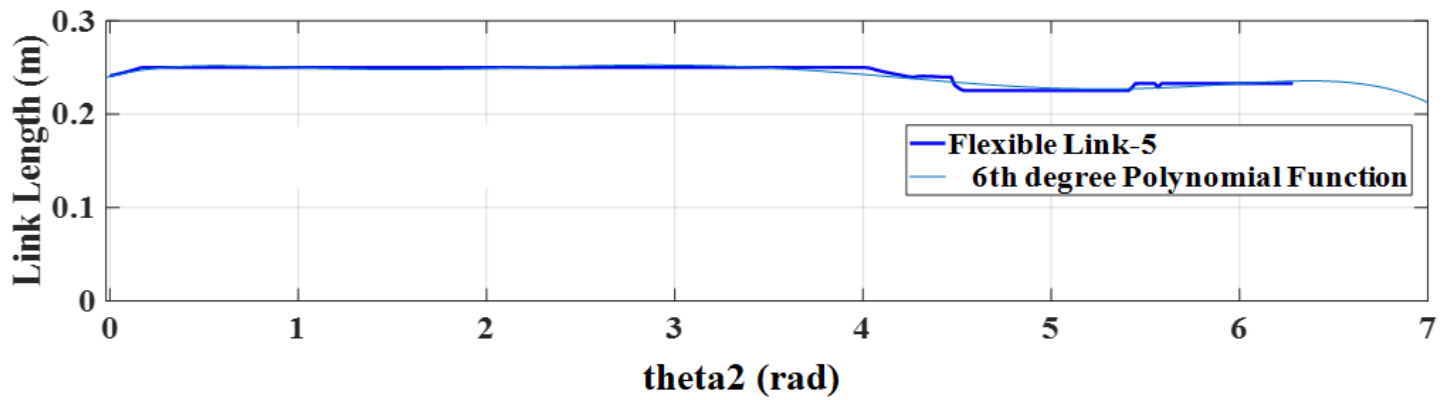

c)
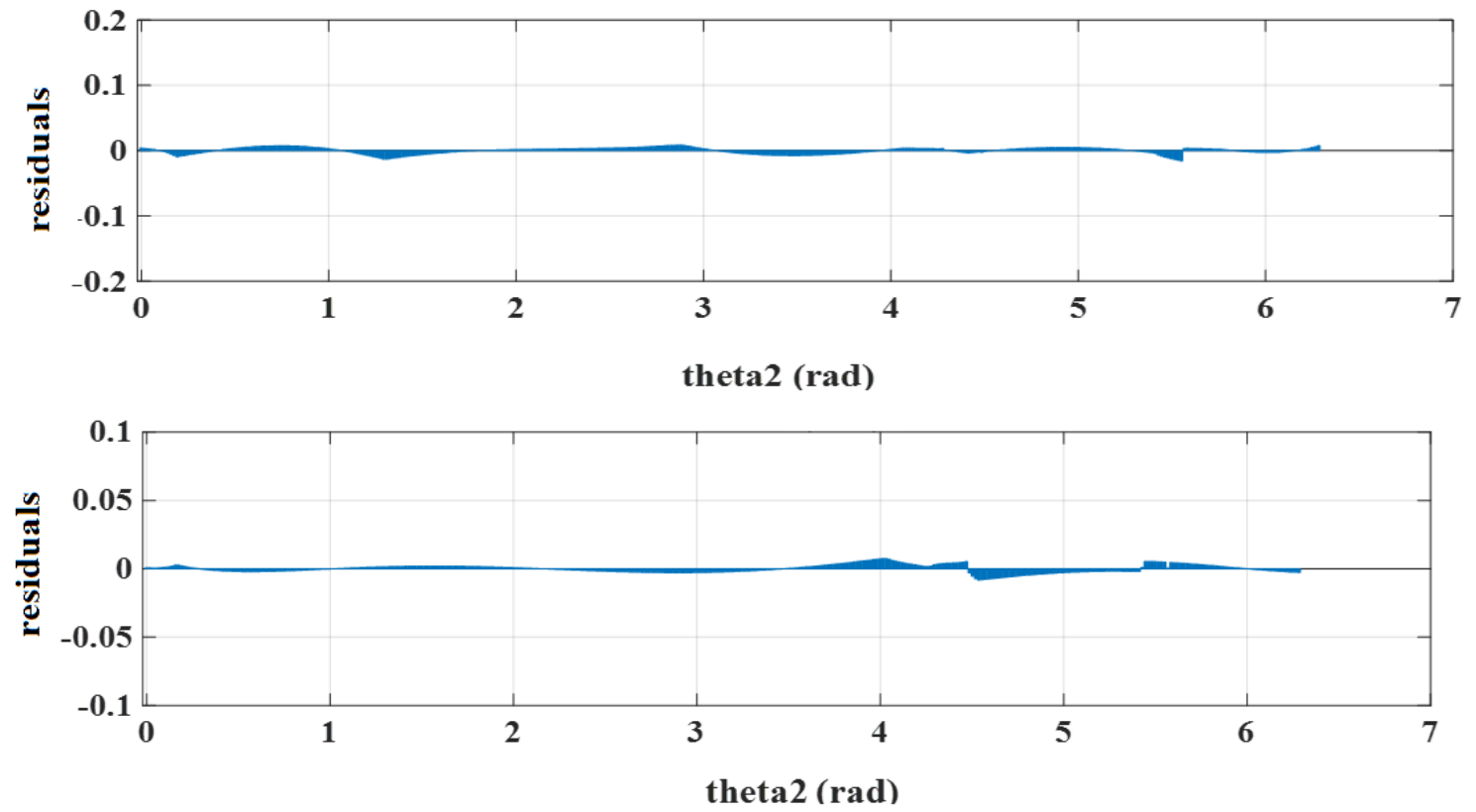

Figure 6. Change of the a) $r_{3}$ and b) $r_{5}$ flexible link lengths w.r.t. $\theta_{2}$ and c) their residuals, respectively.

The related polynomial equations are given in Eq. (16):

$$
\begin{gathered}
r_{3}\left(z\left(\theta_{2}\right)\right)=-0.014 z^{6}-0.0072 z^{5} \\
+0.087 z^{4}+0.016 z^{3}-0.17 z^{2}+0.057 z+0.19, \quad z\left(\theta_{2}\right)=\left(\theta_{2}-3.1\right) / 1.8 \\
r_{5}\left(z\left(\theta_{2}\right)\right)=-0.037 z^{6}+0.0023 z^{5}+0.019 z^{4} \\
-0.005 z^{3}-0.029 z^{2}-0.0074 z+0.25, \quad z\left(\theta_{2}\right)=\left(\theta_{2}-3.1\right) / 1.8
\end{gathered}
$$




\subsubsection{The Simultaneous Constraint Matrix}

Combining 10 kinematic constraint equations (Eq. 7 and Eq. 12) with the 9 dynamic equations (Eq. 14), 19 unknown variables $\left(\left[\ddot{\theta}_{3}, \ddot{r}_{3}, \ddot{\theta}_{5}, \ddot{r}_{5}, a_{c 2, x}, a_{c 2, y}, a_{c 4, x}, a_{c 4, y}, a_{c 6, x}, a_{c 6, y}\right.\right.$, $\left.\left.F_{12, x}, F_{12, y}, \tau_{m}, F_{14, x}, F_{14, y}, \ddot{\theta}_{4}, F_{16, x}, F_{16, y}, \ddot{\theta}_{6}\right]\right)$ are determined by solving the simultaneous constraint matrix equation (Gardner, 2001) as demonstrated in Eq. (17):

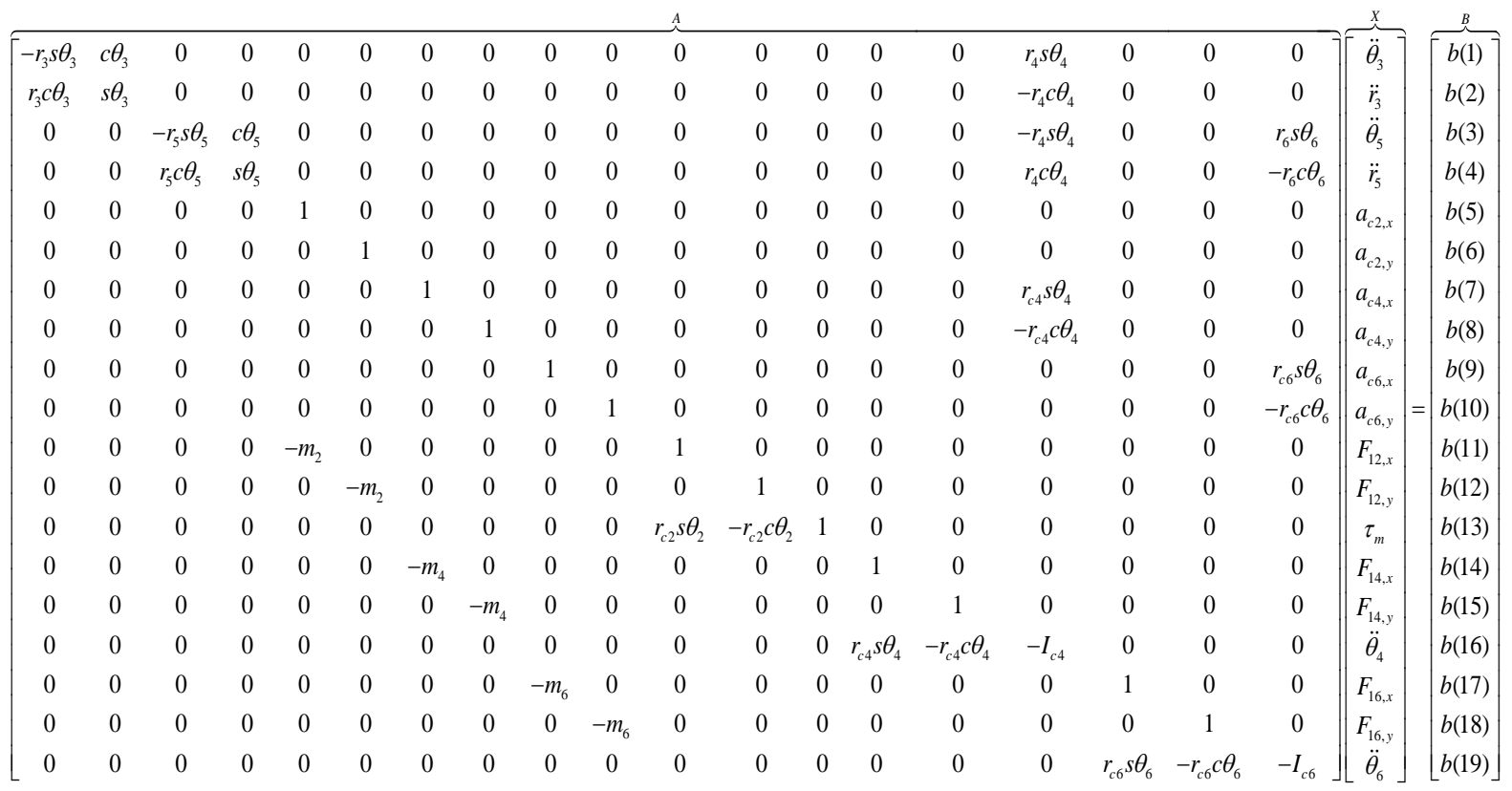

In this matrix, notation $\mathrm{s}$ is for $\sin ($.$) function and \mathrm{c}$ for $\cos ($.$) function.$ 
The notations representing the known vector in Eq. (17) are given in Eq. (18).

$$
\begin{aligned}
& b(1)=r_{3}(\mathrm{t}) \dot{\theta}_{3}(t)^{2} \cos \left(\theta_{3}(t)\right)-r_{4} \dot{\theta}_{4}(t)^{2} \cos \left(\theta_{4}(t)\right)+2 \dot{r}_{3}(\mathrm{t}) \dot{\theta}_{3}(t) \sin \left(\theta_{3}(t)\right) \\
& +r_{2} \dot{\theta}_{2}(t)^{2} \cos \left(\theta_{2}(t)\right)+r_{2} \ddot{\theta}_{2}(t) \sin \left(\theta_{2}(t)\right) b(2)=r_{2} \dot{\theta}_{2}(t)^{2} \sin \left(\theta_{2}(t)\right)+ \\
& r_{3}(\mathrm{t}) \dot{\theta}_{3}(t)^{2} \sin \left(\theta_{3}(t)\right)-2 \dot{r}_{3}(\mathrm{t}) \dot{\theta}_{3}(t) \cos \left(\theta_{3}(t)\right) \\
& -r_{4} \dot{\theta}_{4}(t)^{2} \sin \left(\theta_{4}(t)\right)-r_{2} \ddot{\theta}_{2}(t) \cos \left(\theta_{2}(t)\right) \\
& b(3)=2 \dot{r}_{5}(\mathrm{t}) \dot{\theta}_{5}(t) \sin \left(\theta_{5}(t)\right)+r_{4} \dot{\theta}_{4}(t)^{2} \cos \left(\theta_{4}(t)\right) \\
& +r_{5}(\mathrm{t}) \dot{\theta}_{5}(t)^{2} \cos \left(\theta_{5}(t)\right)-r_{6} \dot{\theta}_{6}(t)^{2} \cos \left(\theta_{6}(t)\right) \\
& b(4)=r_{4} \dot{\theta}_{4}(t)^{2} \sin \left(\theta_{4}(t)\right) \\
& +r_{5}(\mathrm{t}) \dot{\theta}_{5}(t)^{2} \sin \left(\theta_{5}(t)-2 \dot{r}_{5}(\mathrm{t}) \dot{\theta}_{5}(t) \cos \left(\theta_{5}(t)\right)-r_{6} \dot{\theta}_{6}(t)^{2} \sin \left(\theta_{6}(t)\right)\right. \\
& b(5)=-r_{c 2} \dot{\theta}_{2}(t)^{2} \cos \left(\theta_{2}(t)\right)-r_{c 2} \ddot{\theta}_{2}(t) \sin \left(\theta_{2}(t)\right) \\
& b(6)=-r_{c 2} \dot{\theta}_{2}(t)^{2} \sin \left(\theta_{2}(t)\right)+r_{c 2} \ddot{\theta}_{2}(t) \cos \left(\theta_{2}(t)\right) \\
& b(7)=-r_{c 4} \dot{\theta}_{4}(t)^{2} \cos \left(\theta_{4}(t)\right) \\
& b(8)=-r_{c 4} \dot{\theta}_{4}(t)^{2} \sin \left(\theta_{4}(t)\right) \\
& \mathrm{b}(9)=-r_{c 6} \dot{\theta}_{6}(t)^{2} \cos \left(\theta_{6}(t)\right) \\
& b(10)=-r_{c 6} \dot{\theta}_{6}(t)^{2} \sin \left(\theta_{6}(t)\right) \\
& b(11)=-F_{3}(\mathrm{t}) \cos \left(\theta_{3}(t)\right) \\
& b(12)=-F_{3}(\mathrm{t}) \sin \left(\theta_{3}(t)\right)+m_{2} g \\
& b(13)=F_{3}(t) \cos \left(\theta_{3}(t)\right)\left(r_{2}-r_{c 2}\right) \sin \left(\theta_{2}(t)\right) \\
& -F_{3}(t) \sin \left(\theta_{3}(t)\right)\left(r_{2}-r_{c 2}\right) \cos \left(\theta_{2}(t)\right)+I_{c 2} \ddot{\theta}_{2}(t) \\
& b(14)=-F_{5}(t) \cos \left(\theta_{5}(t)\right)-F_{3}(t) \cos \left(\theta_{3}(t)\right) \\
& b(15)=-F_{5}(t) \sin \left(\theta_{5}(t)\right)-F_{3}(t) \sin \left(\theta_{3}(t)\right)+m_{4} g \\
& b(16)=F_{5}(t) \cos \left(\theta_{5}(t)\right)\left(r_{4}-r_{c 4}\right) \sin \left(\theta_{4}(t)\right) \\
& -F_{5}(t) \sin \left(\theta_{5}(t)\right)\left(r_{4}-r_{c 4}\right) \cos \left(\theta_{4}(t)\right) \\
& \left.+F_{3}(t) \cos \left(\theta_{3}(t)\right)\left(r_{4}-r_{c 4}\right) \sin \left(\theta_{4}(t)\right)-F_{3}(t) \sin \left(\theta_{3}(t)\right)\left(r_{4}-r_{c 4}\right) \cos \left(\theta_{4}(t)\right)\right) \\
& b(17)=-F_{5}(t) \cos \left(\theta_{5}(t)\right) \\
& b(18)=-F_{5}(t) \sin \left(\theta_{5}(t)\right)+m_{6} g \\
& b(19)=F_{5}(t) \cos \left(\theta_{5}(t)\right)\left(r_{6}-r_{c 6}\right) \sin \left(\theta_{6}(t)\right) \\
& -F_{5}(t) \sin \left(\theta_{5}(t)\right)\left(r_{6}-r_{c 6}\right) \cos \left(\theta_{6}(t)\right)
\end{aligned}
$$

The unknown vector can be evaluated from the theory of linear algebra by multiplying the inverse of matrix $\mathrm{A}$ (coefficient matrix) and $\mathrm{B}$ (known vector), which implies as $\mathrm{X}=A^{-1} B$. This matrix equation is embedded in a MATLAB function that, in turn, can be embedded into a Simulink-based simulation. 


\section{Simulation and Experimental Results}

\subsection{Experimental Setup}

The experimental setup and the prototype of the flexible Watt six-bar mechanism are demonstrated in this sub-section. The design setup of the Compliant Watt-linkage prototype is demonstrated in Figure 7. This prototype is utilized to contribute to the simulation results. The main structure of the Watt six-bar compliant mechanism is comprised of three rigid links and two flexible beams. The physical properties and their values related to the mechanism are given in Table 2 .

The dimensions of the cross-section of the flexible links are selected from the steel spring strips. The input of the Watt six-bar compliant mechanism is supplied by DC Motor (2.2 Volt at initial, 4 Volt at operating). To predict the movement of the whole compliant mechanism, it should be known how the flexible links buckle. The total length of the flexible links is $440 \mathrm{~mm}$. The first part of the flexible link (coded as 3) is $190 \mathrm{~mm}$, and the second part of the flexible link (coded as 5) is $250 \mathrm{~mm}$ before buckling, respectively. During the operation, the length of flexible links changes while joints that connect the flexible links and rigid links turn. An encoder is attached to each rigid link to measure the link angle and to evaluate the angular crank velocity $\left(\dot{\theta}_{2}(t)\right)$. Even though a DC motor within the house encoder is utilized in the experiments, the DC motor's encoder response was not certain. Therefore, a more accurate encoder is integrated into the motor shaft. The data from the measurements of positions are acquired with an Analogue-Digital Converter (ADC) IOTech personal data acquisition card. Encoders and ADC's are supplied with 6-7 Volt. DC Motor and encoder used in the experiments are presented in Figure 8.

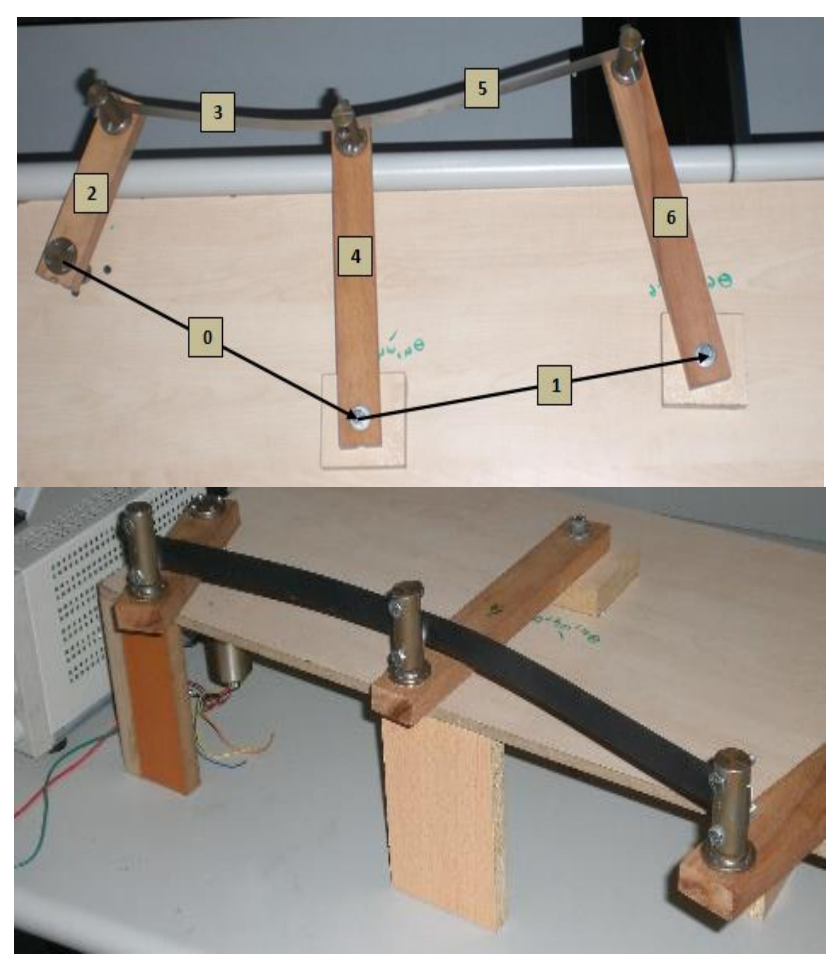

Figure 7. The prototype of the Watt six-bar compliant mechanism. 
Table 2. Physical properties and their values.

\begin{tabular}{|c|c|}
\hline Physical property & Value/Dimension \\
\hline $\begin{array}{l}\text { Length of the flexible } \\
\text { links (pre-buckling } \\
\text { state) }\left(r_{3,0}\right),\left(r_{5,0}\right)\end{array}$ & $19 \mathrm{~cm}, 25 \mathrm{~cm}$ \\
\hline $\begin{array}{l}\text { Length of the rigid links } \\
\left(r_{2}\right),\left(r_{4}\right),\left(r_{6}\right)\end{array}$ & $12 \mathrm{~cm}, 22 \mathrm{~cm}, 24 \mathrm{~cm}$ \\
\hline $\begin{array}{l}\text { Length of the rigid links } \\
\text { (from the lower end to } \\
\text { the centre of gravity) } \\
\left(r_{c 2}\right),\left(r_{c 4}\right),\left(r_{c 6}\right)\end{array}$ & $6 \mathrm{~cm}, 11 \mathrm{~cm}, 12 \mathrm{~cm}$ \\
\hline $\begin{array}{l}\text { Cross-section of the } \\
\text { rigid links }\left(b_{r}{ }^{*} h_{r}\right)\end{array}$ & $36 \mathrm{~mm}$ * $18 \mathrm{~mm}$ \\
\hline $\begin{array}{l}\text { Mass of the rigid links } \\
\qquad\left(m_{2}\right)\left(m_{4}\right)\left(m_{6}\right)\end{array}$ & $\begin{array}{l}0.0389 \mathrm{~kg}, 0.0713 \\
\mathrm{~kg}, 0.0778 \mathrm{~kg}\end{array}$ \\
\hline $\begin{array}{l}\text { Moment of inertia of the } \\
\text { rigid links } \\
\left(I_{c 2}\right),\left(I_{c 4}\right),\left(I_{c 6}\right)\end{array}$ & $1.7496^{*} 10^{-8} \mathrm{kgm}^{2}$ \\
\hline $\begin{array}{l}\text { Cross-section of the } \\
\text { flexible links }\left(b_{f}^{*} h_{f}\right)\end{array}$ & $21 \mathrm{~mm} * 0.2 \mathrm{~mm}$ \\
\hline $\begin{array}{l}\text { Young's modulus of the } \\
\text { flexible links } \\
\left(E_{3}\right),\left(E_{5}\right)\end{array}$ & $2.07^{*} 10^{11} \mathrm{~N} / \mathrm{m}^{2}$ \\
\hline $\begin{array}{l}\text { Ground link length } \\
\qquad\left(r_{1 \alpha}\right),\left(r_{1 \beta}\right)\end{array}$ & $0.3 \mathrm{~m}$ \\
\hline $\begin{array}{l}\text { Ground link orientation } \\
\qquad\left(\theta_{1 \alpha}\right),\left(\theta_{1 \beta}\right)\end{array}$ & $\begin{array}{l}25^{\circ},-10^{\circ} \\
\text { counterclockwise } \\
\text { rotation }(+)\end{array}$ \\
\hline
\end{tabular}

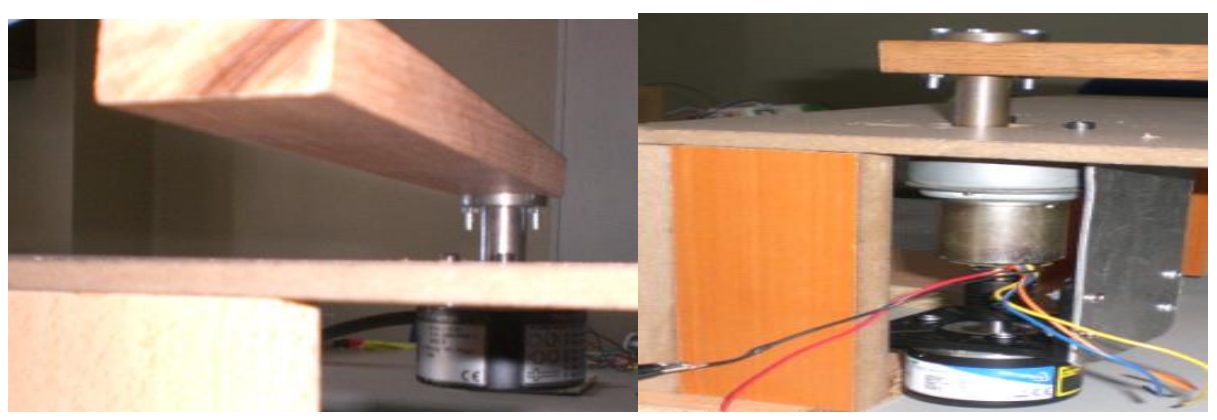

Figure 8. DC motor and encoder used in the experiment.

Data processing is conducted by MATLAB/SIMULINK software. The data acquired from encoders are processed in MATLAB/SIMULINK to acquire the angular velocity and angular 
position of the rigid links. The Simulink block diagram utilized for data processing is represented in Figure 9.
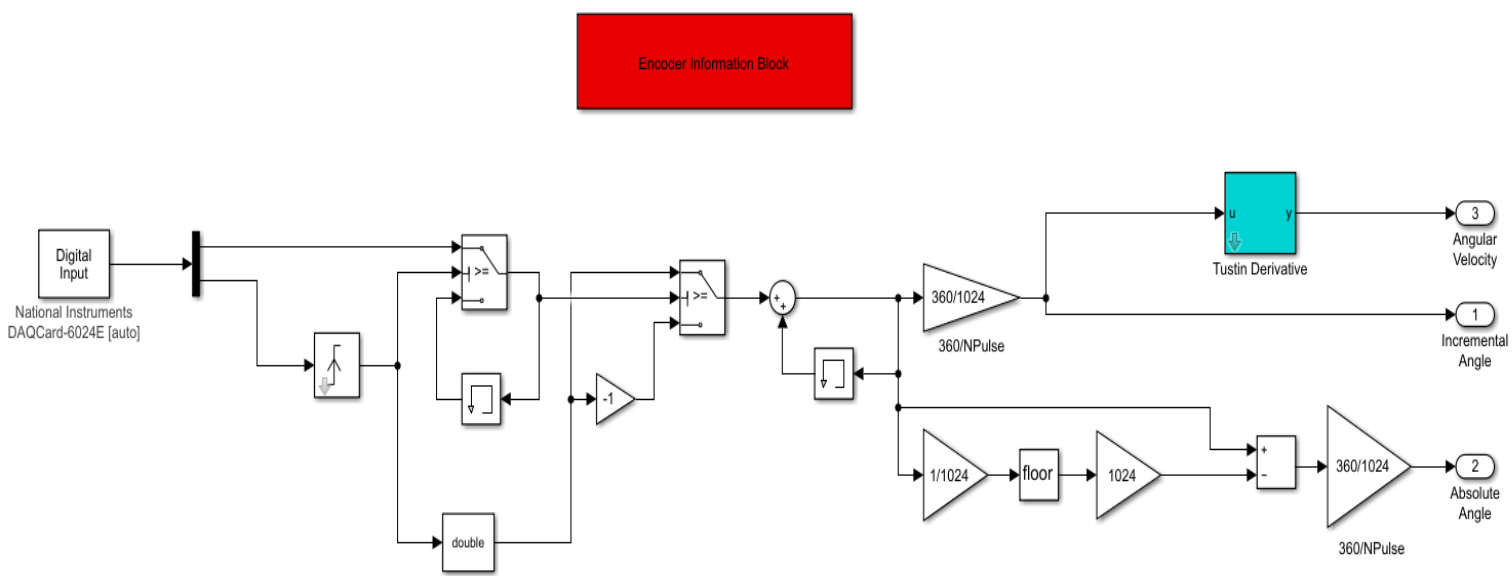

Figure 9. Simulink block diagram utilized for data processing.

The angular velocity of each rigid link is obtained from the angular displacement data of the related encoder. Unwanted noise is reduced by passing the collected raw data through differential operator and filter, respectively, given in Eq. (19):

$$
D(.)=\frac{d(V(t))}{d t} \approx \frac{s}{\tau s+1} V(s), \quad F(s)=\frac{\omega_{n}^{2}}{s^{2}+2 \xi \omega_{n} s+\omega_{n}^{2}}
$$

The differential operator guarantees the causality. $\tau$ is selected as 120.51 . The filter has a cutoff frequency at $\omega_{n}=10 \mathrm{rad} / \mathrm{sec}$. $\xi$ is selected as 0.707 . The filter parameters were chosen to obtain a continuous noise-free response of the angular velocity of each rigid link.

\subsection{Experimental and Simulation Results}

Link-2 has an initial angular velocity of $1.57 \mathrm{rad} / \mathrm{s}$ and an initial position of $270^{\circ}$. The other initial conditions (configurations) were evaluated by conducting quasi-static analysis and given in Table 3 .

Table 3. Initial configuration of the mechanism.

\begin{tabular}{|c|c|}
\hline$\theta_{3,0}$ & $0.3345 \mathrm{rad}$ \\
\hline$\theta_{4,0}$ & $4.1888 \mathrm{rad}$ \\
\hline$\theta_{5,0}$ & $6.1979 \mathrm{rad}$ \\
\hline$\theta_{6,0}$ & $3.8582 \mathrm{rad}$ \\
\hline$r_{3,0}$ & $0.1714 \mathrm{~m}$ \\
\hline$r_{5,0}$ & $0.2253 \mathrm{~m}$ \\
\hline
\end{tabular}

The simulations were done in MATLAB/SIMULINK program. When Link-2 turns one cycle $\left[270^{\circ}-\left(-90^{\circ}\right)\right]$, the changes in angular positions $\left(\theta_{3}, \theta_{4}, \theta_{5}, \theta_{6}\right)$, angular velocities $\left(\dot{\theta}_{3}, \dot{\theta}_{4}, \dot{\theta}_{5}, \dot{\theta}_{6}\right)$ 
and angular accelerations $\left(\ddot{\theta}_{3}, \ddot{\theta}_{4}, \ddot{\theta}_{5}, \ddot{\theta}_{6}\right)$ w.r.t. time are presented in Figure 10a-Figure 10d, Figure 11a-Figure 11d, Figure 12a-Figure 12d, respectively.

a)

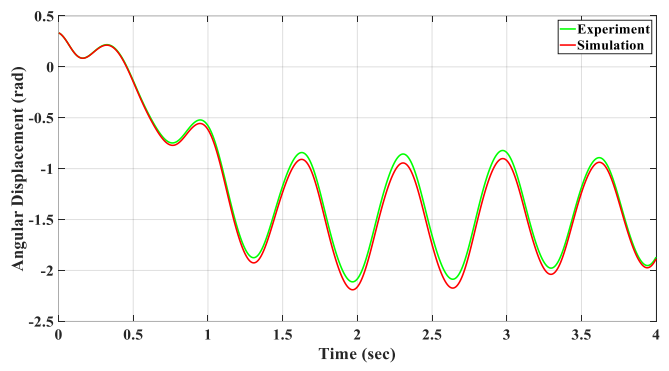

c)

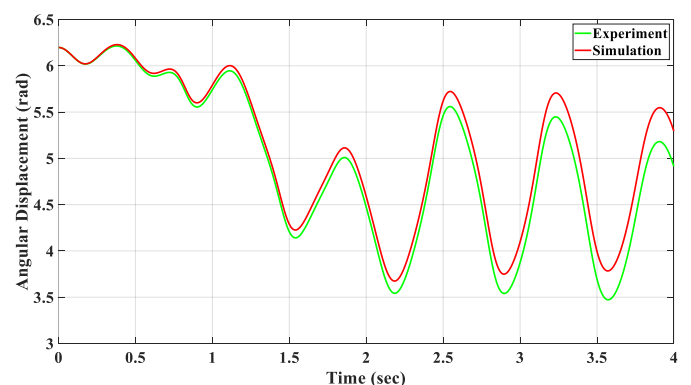

b)

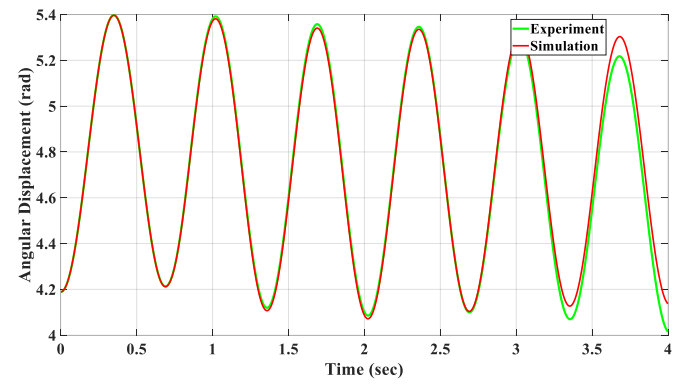

d)

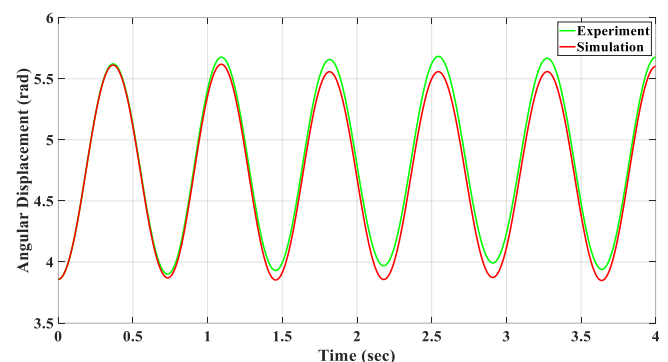

Figure 10. Angular displacement trajectory. a) $\theta 3$-time graph, b) $\theta 4$-time graph, c) $\theta 5$-time graph d) $\theta 6$-time graph

a)

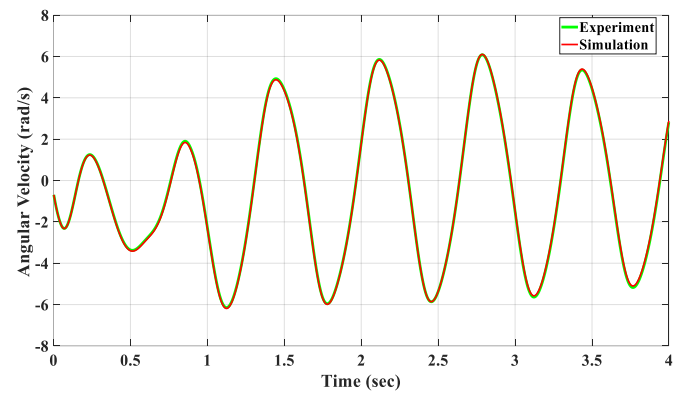

c)

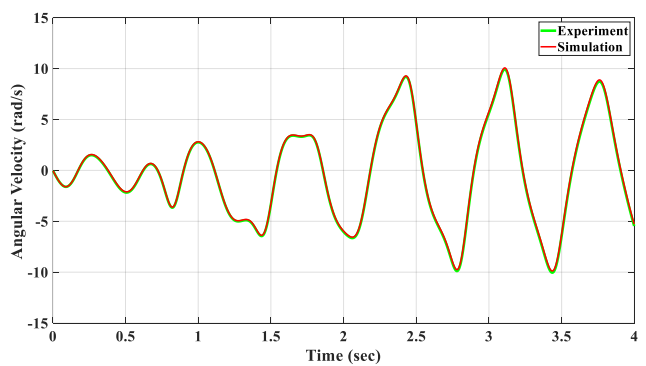

b)

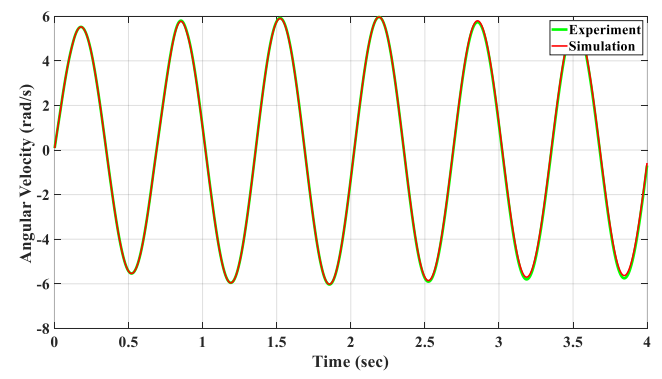

d)

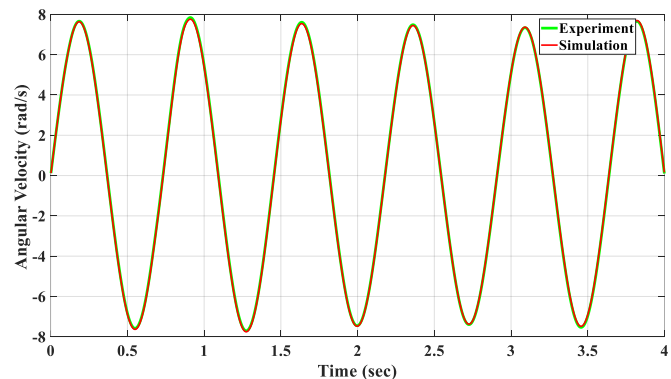

Figure 11. Angular velocity trajectory. a) w3-time graph, b) w4-time graph, c) w5-time graph, d) w6-time graph 
a)

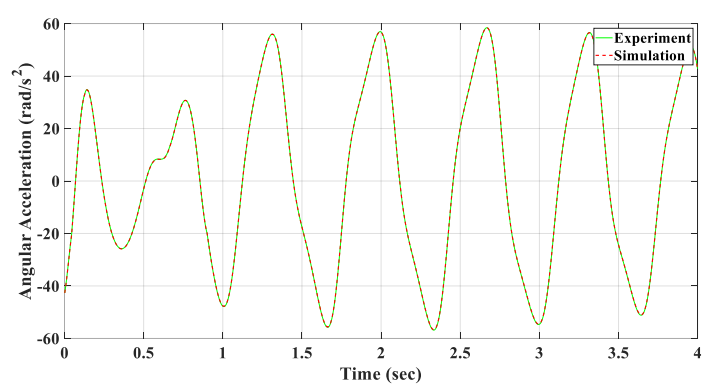

c)

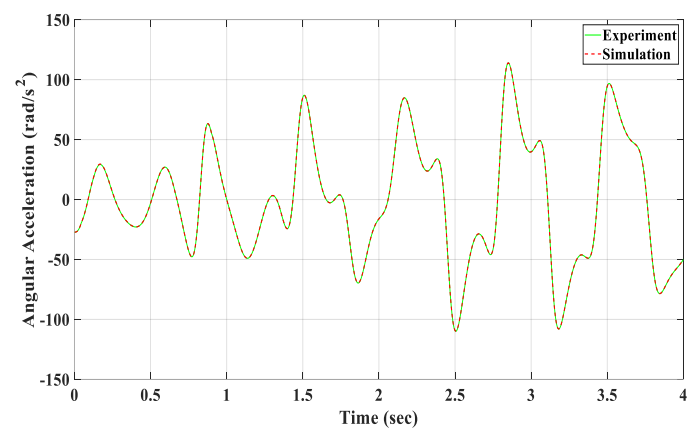

b)

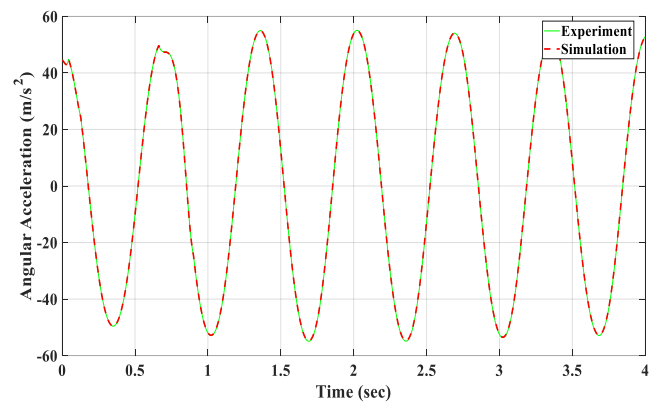

d)

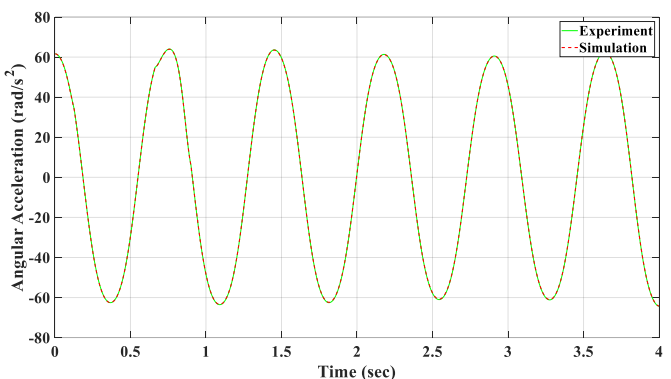

Figure 12. Angular acceleration trajectory. a) a3-time graph, b) a4-time graph, c) a5-time graph, d) a6-time graph

The reaction forces $\left(F_{12, x}, F_{12, y}, F_{14, x}, F_{14, y}, F_{16, x}, F_{16, y}\right)$, motor torque $\left(\tau_{m}\right)$ are represented in Figure 13a-Figure 13b, respectively.

a)

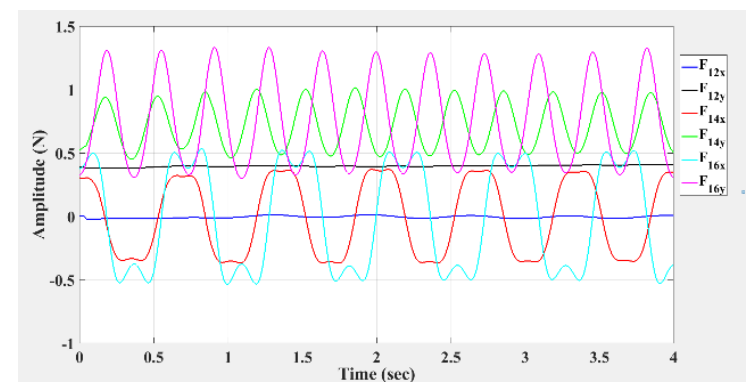

b)

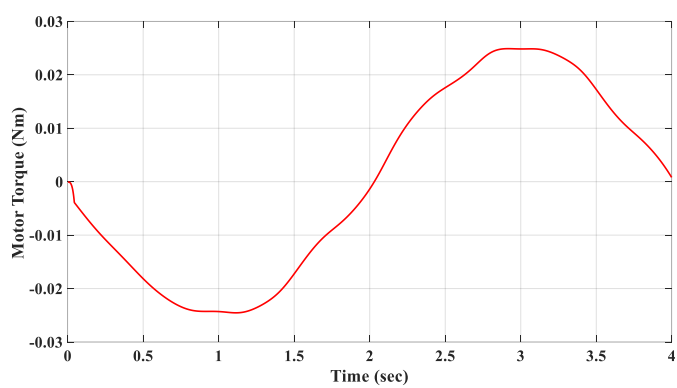

Figure 13. Kinetic trajectory. a) Reaction force-time, b) Motor torque-time

One can deduce from the results that the kinematic and kinetic trajectories of the mechanism were experimentally validated. The deformation of the flexible components are evident and varies in the motion cycle of the mechanism. 


\section{Conclusions}

This paper describes the design procedure and dynamic analysis of a partially compliant Watt six-bar linkage combining the PRBM and elastica buckling theory. Polynomial curve fits of the pinned-pinned flexible beam and the PRBM of the large deflecting cantilever beam are synthesized in dynamic simulation via simultaneous constraint matrix. The kinematic analysis is realized through vector loop closure, the force equilibrium of elastic elements, and numerically solving the nonlinear dynamical equations. The 4th and 6th link curves are symmetric. The path of the mechanism is almost straight in one section and completely parallel. Therefore, the compliant Watt six-bar linkage is quite suitable for legged robot design. The parallel motion of the joints leads to an extended contact surface of the leg coupled with the terrain. The balance of the legs can be sustained without damaging effects on the terrain. The number of degrees of freedom is reduced to one so that the control system synthesis and motion analysis are easily conducted. The full dynamic simulation results are verified by comparing the experimental results. The synergy of the PRBM and elastica buckling theory serves as an effective method in the analysis and synthesis of new compliant mechanism designs. The compliant synthesis approach for industrial implementations wherein accuracy is extremely important such as medical, welding and manufacturing robot cause compliant modelling wherein failures are considered.

\section{Nomenclature}

$r_{1 \alpha} \quad$ Ground link length $(\alpha)$

$\theta_{1 \alpha} \quad$ Ground link orientation $(\alpha)$

$r_{1 \beta} \quad$ Ground link length $(\beta)$

$\theta_{1 \beta} \quad$ Ground link orientation $(\beta)$

$r_{i} \quad i^{\text {th }}$ flexible link length $(i=3,5)$

$r_{i, 0} \quad i^{\text {th }}$ flexible link initial length (prebuckling state)

$\dot{r}_{i} \quad i^{\text {th }}$ flexible link buckling velocity

$\ddot{r}_{i} \quad i^{\text {th }}$ flexible link buckling acceleration

$\vartheta_{i} \quad i^{\text {th }}$ flexible link deflection

$\theta_{i} \quad i^{\text {th }}$ flexible link angle

$\dot{\theta}_{i} \quad i^{\text {th }}$ flexible link angular velocity

$\ddot{\theta}_{i} \quad i^{\text {th }}$ flexible link angular acceleration

$F_{i} \quad i^{\text {th }}$ flexible link spring force

$b_{f, i} \quad i^{\text {th }}$ flexible link cross-sectional width

$h_{f, i} \quad i^{t h} \quad$ flexible link cross-sectional thickness

$E I_{i} \quad i^{\text {th }}$ flexible link flexural rigidity

$r_{j} \quad j^{\text {th }}$ rigid link length $(j=2,4,6)$

$\theta_{j} \quad j^{\text {th }}$ rigid link angle

$\dot{\theta}_{j} \quad j^{\text {th }}$ rigid link angular velocity

$\ddot{\theta}_{j} \quad j^{\text {th }}$ rigid link angular acceleration

$b_{r, j} \quad j^{\text {th }}$ rigid link cross-sectional width

$h_{r, j} \quad i^{\text {th }}$ rigid link cross-sectional thickness

$F_{i j, x} \quad$ The horizontal force of link $i$ acting on the link $j$
$F_{i j, y} \quad$ The vertical force of link $i$ acting on the link $j$

$C_{O G} \quad$ Center of gravity of the $j^{\text {th }}$ link

$r_{\mathrm{cj}}$ Length from $(j-$ 1) $)^{\text {th }}$ joint to $j^{\text {th }} \operatorname{COG}_{j}$

$m_{j} \quad j^{\text {th }}$ rigid link mass

$I_{c j} \quad j^{\text {th }}$ rigid link moment of inertia about the $C O G_{j}$

$g \quad$ Acceleration due to gravity

$\tau_{m} \quad$ Applied DC-motor torque 


\section{Author Statement}

The authors confirm contribution to the paper as follows: Study conception, system design, analysis and data interpretation are done by Çağlar Uyulan. Experimental setup and data acquisition are done by Batuhan İpek. Draft manuscript is prepared by Çağlar Uyulan. All authors reviewed the results and approved the final version of the manuscript.

\section{Conflict of Interest}

The authors declare no conflict of interest.

\section{References}

Bapat, G. M., \& Sujatha, S. (2017). A Method for Optimal Synthesis of a Biomimetic Four-Bar Linkage Knee Joint for a Knee-Ankle-Foot Orthosis. Journal of Biomimetics, Biomaterials and Biomedical 20-28. doi:10.4028/www.scientific.net/jbbbe.32.20

Berselli, G., Meng, Q., Vertechy, R., \& Castelli, V. P. (2015). An improved design method for the dimensional synthesis of flexure-based compliant mechanisms: Optimization procedure and experimental validation. Meccanica, 51(5), 1209-1225. doi:10.1007/s11012-015-0276-z

Bishopp, K. E., \& Drucker, D. C. (1945). Large deflection of cantilever beams. Quarterly of Applied Mathematics, 3(3), 272-275.

Chen, F., \& Huang, H. (2005). The Use Of Fuzzy Logic In The Taguchi Method For The Determination Of The Tolerance Of A Six-Bar Hinge Mechanism. Transactions of the Canadian Society for Mechanical Engineering, 29(3), 477-489. doi:10.1139/tcsme-20050029

Chen, F., \& Tzeng, Y. (2006). A Simple Approach For The Tolerance Design Of Watt-I Type Six-Bar Hinge Mechanism Using Taguchi Method. International Journal of Reliability, Quality and Safety Engineering, 13(02), 157-169. doi:10.1142/s0218539306002185

Eren, R., \& Aydemir, A. (2004). An Approach to Kinematic Design of Four-bar Sley Drive Mechanisms in Weaving. The Journal of The Textile Institute, 95(1-6), 193-205. doi:10.1533/joti.2002.0055

Gardner, J. F. (2001). Simulations of machines using MATLAB and Simulink. California: Wadsworth-Thomson Learning.

Gezgin, E., Chang, P., \& Akhan, A. F. (2016). Synthesis of a Watt II six-bar linkage in the design of a hand rehabilitation robot. Mechanism and Machine Theory, 104, 177-189. doi:10.1016/j.mechmachtheory.2016.05.023

Ghosh, A., \& Corves, B. (2015). Microfabrication and Futuristic Issues. Introduction to Micromechanisms and Microactuators Mechanisms and Machine Science, 133-153. doi:10.1007/978-81-322-2144-9_8 
Gouker, R. M., Gupta, S. K., Bruck, H. A., \& Holzschuh, T. (2006). Manufacturing of multimaterial compliant mechanisms using multi-material molding. The International Journal of Advanced Manufacturing Technology, 30(11-12), 1049-1075. doi:10.1007/s00170-005-0152-4

Her, I., \& Midha, A. (1987). A Compliance Number Concept for Compliant Mechanisms, and Type Synthesis. Journal of Mechanisms, Transmissions, and Automation in Design, 109(3), 348-355. doi:10.1115/1.3258802

Howell, L. L., \& Midha, A. (1994). A Generalized Loop-Closure Theory for the Analysis and Synthesis of Compliant Mechanisms. 23rd Biennial Mechanisms Conference: Machine Elements and Machine Dynamics. doi:10.1115/detc1994-0293

Howell, L. L., \& Midha, A. (1994). A Method for the Design of Compliant Mechanisms With Small-Length Flexural Pivots. Journal of Mechanical Design, 116(1), 280-290. doi:10.1115/1.2919359

Howell, L. L., Magleby, S. P., \& Olsen, B. M. (2013). Handbook of compliant mechanisms. Chichester, West Sussex, United Kingdom: John Wiley \& Sons.

Jaiswal, A., \& Jawale, H. P. (2018). Synthesis and optimization of four bar mechanism with six design parameters. doi:10.1063/1.5029590

Jin, D., Zhang, R., Dimo, H., Wang, R., \& Zhang, J. (2003). Kinematic and dynamic performance of prosthetic knee joint using six-bar mechanism. The Journal of Rehabilitation Research and Development, 40(1), 39. doi:10.1682/jrrd.2003.01.0039

Jovanova, J., \& Frecker, M. (2017). Two Stage Design of Compliant Mechanisms With Superelastic Compliant Joints. Volume 2: Modeling, Simulation and Control of Adaptive Systems; Integrated System Design and Implementation; Structural Health Monitoring. doi:10.1115/smasis2017-3825

Khemili, I., Abdallah, M. A., \& Aifaoui, N. (2018). Multi-objective optimization of a flexible slider-crank mechanism synthesis, based on dynamic responses. Engineering Optimization, 51(6), 978-999. doi:10.1080/0305215x.2018.1508574

Khosraviani, K., \& Leung, A. M. (2012). Robust micromachining of compliant mechanisms using silicides. Journal of Micromechanics and Microengineering, 23(1), 015015. doi:10.1088/0960-1317/23/1/015015

Konopasek, M. (1980). Classical Elastica Theory and its Generalizations. Mechanics of Flexible Fibre Assemblies, 255-274. doi:10.1007/978-94-011-9774-8_14

Lefebvre, T., Xiao, J., Bruyninckx, H., \& Gersem, G. D. (2005). Active compliant motion: A survey. Advanced Robotics, 19(5), 479-499. doi:10.1163/156855305323383767

Linß, S., Henning, S., \& Zentner, L. (2019). Modeling and Design of Flexure Hinge-Based Compliant Mechanisms. Kinematics - Analysis and Applications. doi:10.5772/intechopen.85224 
Liu, Y., \& Mcphee, J. (2004). Automated Type Synthesis of Planar Mechanisms Using Numeric Optimization With Genetic Algorithms. Journal of Mechanical Design, 127(5), 910-916. doi:10.1115/1.1904049

Lobontiu, N. (2014). Compliance-based matrix method for modeling the quasi-static response of planar serial flexure-hinge mechanisms. Precision Engineering, 38(3), 639-650. doi:10.1016/j.precisioneng.2014.02.014

Luo, Y., Liu, W., \& Wu, L. (2015). Analysis of the displacement of lumped compliant parallelguiding mechanism considering parasitic rotation and deflection on the guiding plate and rigid beams. Mechanism and Machine Theory, 91, 50-68. doi:10.1016/j.mechmachtheory.2015.04.007

Luo, Z. (2006). Theoretical And Algorithmic On Topology Optimization Design Of Distributed Compliant Mechanisms. Chinese Journal of Mechanical Engineering, 42(10), 27. doi:10.3901/jme.2006.10.027

Luo, Z., Shang, J., Wei, G., \& Ren, L. (2018). A reconfigurable hybrid wheel-track mobile robot based on Watt II six-bar linkage. Mechanism and Machine Theory, 128, 16-32. doi:10.1016/j.mechmachtheory.2018.04.020

Midha, A., Howell, L. L., \& Norton, T. W. (2000). Limit positions of compliant mechanisms using the pseudo-rigid-body model concept. Mechanism and Machine Theory, 35(1), 99-115. doi:10.1016/s0094-114x(98)00093-7

Mirth, J. A. (2012). The Application of Geometric Constraint Programming to the Design of Motion Generating Six-Bar Linkages. Volume 4: 36th Mechanisms and Robotics Conference, Parts A and B. doi:10.1115/detc2012-70176

Mirth, J. A., \& Chase, T. R. (1993). Circuit Analysis of Watt Chain Six-Bar Mechanisms. Journal of Mechanical Design, 115(2), 214-222. doi:10.1115/1.2919180

Niknam, A., \& Farhang, K. (2018). Vibration Instability in a Large Motion Bistable Compliant Mechanism Due to Stribeck Friction. Journal of Vibration and Acoustics, 140(6). doi:10.1115/1.4040513

Pavlovic, N., \& Pavlovic, N. (2005). Mobility of the compliant joints and compliant mechanisms. Theoretical and Applied Mechanics Teorijska I Primenjena Mehanika, 32(4), 341-357. doi:10.2298/tam0504341p

Pieber, M., \& Gerstmayr, J. (2020). Six-Bar Linkages With Compliant Mechanisms for an Adaptive Robot. Volume 10: 44th Mechanisms and Robotics Conference (MR). doi:10.1115/detc2020-22546

Plecnik, M. M., \& Mccarthy, J. M. (2014). Vehicle Suspension Design Based on a Six-Bar Linkage. Volume 5A: 38th Mechanisms and Robotics Conference. doi:10.1115/detc2014-35374 
Plecnik, M., Mccarthy, J. M., \& Wampler, C. W. (2014). Kinematic Synthesis of a Watt I Six-Bar Linkage for Body Guidance. Advances in Robot Kinematics, 317-325. doi:10.1007/9783-319-06698-1_33

Primrose, E. J., Freudenstein, F., \& Roth, B. (1967a). Six-bar motion III. Extension of the six-bar techniques to eight-bar and 2n-bar mechanisms. Archive for Rational Mechanics and Analysis, 24(1), 73-77. doi:10.1007/bf00251593

Primrose, E. J., Freudenstein, F., \& Roth, B. (1967b). Six-bar motion I. The Watt mechanism. Archive for Rational Mechanics and Analysis, 24(1), 22-41. doi:10.1007/bf00251591

Prosser, D., Basrai, T., Dickert, J., Ratti, J., Crassidis, A., \& Vachtsevanos, G. (2011). Wing kinematics and aerodynamics of a hovering flapping Micro Aerial Vehicle. 2011 Aerospace Conference. doi:10.1109/aero.2011.5747521

Ramirez, I. A., \& Lusk, C. P. (2011). Spatial-Beam Large-Deflection Equations and PseudoRigid-Body Model for Axisymmetric Cantilever Beams. Volume 6: 35th Mechanisms and Robotics Conference, Parts A and B. doi:10.1115/detc2011-47389

Sonawale, K. H., Arredondo, A., \& Mccarthy, J. M. (2013). Computer Aided Design of Useful Spherical Watt I Six-Bar Linkages. Volume 6A: 37th Mechanisms and Robotics Conference. doi:10.1115/detc2013-13454

Sönmez, Ü, \& Tutum, C. C. (2008). A Compliant Bistable Mechanism Design Incorporating Elastica Buckling Beam Theory and Pseudo-Rigid-Body Model. Journal of Mechanical Design, 130(4). doi:10.1115/1.2839009

Sönmez, Ü. (2000). Compliant Mechanism Design and Synthesis Using Buckling and SnapThrough Buckling of Flexible Members, PhD thesis, The Pennsylvania State University, State College.

Tsuge, B. Y., \& Mccarthy, J. M. (2014). Fitting Useful Planar Four-Bar and Six-Bar Linkages to Over-Specified Tasks. Interdisciplinary Applications of Kinematics Mechanisms and Machine Science, 171-178. doi:10.1007/978-3-319-10723-3_18

Venkiteswaran, V. K., \& Su, H. (2016). Extension Effects in Compliant Joints and Pseudo-RigidBody Models. Journal of Mechanical Design, 138(9). doi:10.1115/1.4034111

Wang, J., Ting, K., Zhao, D., Wang, Q., Sun, J., You, Y., \& Nie, L. (2014). Full Rotatability of Watt Six-Bar Linkages. Volume 5A: 38th Mechanisms and Robotics Conference. doi:10.1115/detc2014-34207

Wang, M. Y., \& Chen, S. (2009). Compliant Mechanism Optimization: Analysis and Design with Intrinsic Characteristic Stiffness. Mechanics Based Design of Structures and Machines, 37(2), 183-200. doi:10.1080/15397730902761932 
Xi, Y., Xia, Y., Li, X., Sun, Y., \& Wang, H. (2010). Kinematic analysis and parameter optimization of six-bar linkage based on ADAMS. 2010 International Conference on Mechanic Automation and Control Engineering. doi:10.1109/mace.2010.5536443

Yi, L. \& Leinonen, T. (2003). Computer Simulation of Path and Motion Generation With SixBar Linkage. Volume 2: 29th Design Automation Conference, Parts A and B. doi:10.1115/detc2003/dac-48831

Yu, Y., Zhou, P., \& Xu, Q. (2018). Kinematic and dynamic analysis of compliant mechanisms considering both lateral and axial deformations of flexural beams. Proceedings of the Institution of Mechanical Engineers, Part C: Journal of Mechanical Engineering Science, 233(3), 1007-1020. doi:10.1177/0954406218760956

Zhang, A., \& Chen, G. (2012). A Comprehensive Elliptic Integral Solution to the Large Deflection Problems of Cantilever Beams in Compliant Mechanisms. Volume 4: 36th Mechanisms and Robotics Conference, Parts A and B. doi:10.1115/detc2012-70239

Zhang, X., \& Zhu, B. (2018). Topology Optimization of Distributed Compliant Mechanisms. Topology Optimization of Compliant Mechanisms, 81-119. doi:10.1007/978-981-13-0432-3_3

Zhang, Z., Chang, B., Zhao, J., Yang, Q., \& Liu, X. (2020). Design, Optimization, and Experiment on a Bioinspired Jumping Robot with a Six-Bar Leg Mechanism Based on Jumping Stability. Mathematical Problems in Engineering, 2020, 1-23. doi:10.1155/2020/3507203

Zu, Y. L., \& Wei, J. (2013). Simulation and Optimization Design of Inversion Six-Bar Linkage on Loader. Applied Mechanics and Materials, 416-417, 1822-1825. doi:10.4028/www.scientific.net/amm.416-417.1822 